\title{
MID-INFRARED SELECTION OF ACTIVE GALACTIC NUCLEI WITH THE WIDE-FIELD INFRARED SURVEY EXPLORER. II. PROPERTIES OF WISE-SELECTED ACTIVE GALACTIC NUCLEI IN THE NDWFS BOÖTES FIELD
}

\author{
R. J. Assef ${ }^{1,14}$, D. Stern ${ }^{1}$, C. S. Kochanek ${ }^{2,3}$, A. W. Blain ${ }^{4}$, M. Brodwin ${ }^{5}$, M. J. I. Brown ${ }^{6}$, E. Donoso ${ }^{7,8}$, \\ P. R. M. EisenhardT ${ }^{1}$, B. T. Jannuzi ${ }^{9}$, T. H. JarretT ${ }^{7}, 10$, S. A. Stanford ${ }^{11,12}$, C.-W. Tsai ${ }^{1}, 14$, J. Wu ${ }^{13}$, And L. Yan ${ }^{7}$ \\ ${ }^{1}$ Jet Propulsion Laboratory, California Institute of Technology, 4800 Oak Grove Drive, Mail Stop 169-236, Pasadena, CA 91109, USA; roberto.j.assef@jpl.nasa.gov \\ ${ }^{2}$ Department of Astronomy, The Ohio State University, 140 West 18th Avenue, Columbus, OH 43210, USA \\ ${ }^{3}$ The Center for Cosmology and Astroparticle Physics, The Ohio State University, 191 West Woodruff Avenue, Columbus, OH 43210, USA \\ ${ }^{4}$ Physics \& Astronomy, University of Leicester, 1 University Road, Leicester, LE1 7RH, UK \\ ${ }^{5}$ Department of Physics, University of Missouri, 5110 Rockhill Road, Kansas City, MO 64110, USA \\ ${ }^{6}$ School of Physics, Monash University, Clayton 3800, Victoria, Australia \\ ${ }^{7}$ Infrared Processing and Analysis Center, California Institute of Technology, Pasadena, CA 91125, USA \\ ${ }^{8}$ Instituto de Ciencias Astronomicas, de la Tierra, y del Espacio (ICATE), 5400 San Juan, Argentina \\ ${ }^{9}$ Steward Observatory, University of Arizona, Tucson, AZ 85721, USA \\ ${ }^{10}$ Astronomy Department, University of Cape Town, Rondebosch, South Africa \\ ${ }^{11}$ Department of Physics, University of California, One Shields Avenue, Davis, CA 95616, USA \\ ${ }^{12}$ Institute of Geophysics and Planetary Physics, Lawrence Livermore National Laboratory, Livermore, CA 94550, USA \\ ${ }^{13}$ UCLA Astronomy, P.O. Box 951547, Los Angeles, CA 90095-1547, USA \\ Received 2012 September 25; accepted 2013 May 22; published 2013 July 2
}

\begin{abstract}
Stern et al. presented a study of Wide-field Infrared Survey Explorer (WISE) selection of active galactic nuclei (AGNs) in the $2 \mathrm{deg}^{2}$ COSMOS field, finding that a simple criterion $\mathrm{W} 1-\mathrm{W} 2 \geqslant 0.8$ provides a highly reliable and complete AGN sample for $\mathrm{W} 2<15.05$, where the $\mathrm{W} 1$ and $\mathrm{W} 2$ passbands are centered at $3.4 \mu \mathrm{m}$ and $4.6 \mu \mathrm{m}$, respectively. Here we extend this study using the larger $9 \mathrm{deg}^{2}$ NOAO Deep Wide-Field Survey Boötes field which also has considerably deeper WISE observations than the COSMOS field, and find that this simple color cut significantly loses reliability at fainter fluxes. We define a modified selection criterion combining the W1-W2 color and the W2 magnitude to provide highly reliable or highly complete AGN samples for fainter WISE sources. In particular, we define a color-magnitude cut that finds $130 \pm 4 \mathrm{deg}^{-2}$ AGN candidates for W2 $<17.11$ with 90\% reliability. Using the extensive UV through mid-IR broadband photometry available in this field, we study the spectral energy distributions of WISE AGN candidates. We find that, as expected, the WISE AGN selection can identify highly obscured AGNs, but that it is biased toward objects where the AGN dominates the bolometric luminosity output. We study the distribution of reddening in the AGN sample and discuss a formalism to account for sample incompleteness based on the step-wise maximum-likelihood method of Efstathiou et al. The resulting dust obscuration distributions depend strongly on AGN luminosity, consistent with the trend expected for a receding torus. At $L_{\mathrm{AGN}} \sim 3 \times 10^{44} \mathrm{erg} \mathrm{s}^{-1}, 29 \% \pm 7 \%$ of AGNs are observed as Type 1, while at $\sim 4 \times 10^{45} \mathrm{erg} \mathrm{s}^{-1}$ the fraction is $64 \% \pm 13 \%$. The distribution of obscuration values suggests that dust in the torus is present as both a diffuse medium and in optically thick clouds.
\end{abstract}

Key words: galaxies: active - methods: statistical - quasars: general

Online-only material: color figures, machine-readable table

\section{INTRODUCTION}

Active galactic nuclei (AGNs) have been proposed to play an important role in several aspects of galaxy evolution, such as quenching star formation in their host galaxies by heating and/or mechanically pushing their gas reservoirs into the intergalactic medium (IGM; see, e.g., Hopkins et al. 2005), preventing cooling flows at the center of galaxy clusters (see, e.g., Croton et al. 2006), and possibly by contributing significantly to the reionization of the universe at high redshift (Glikman et al. 2010, 2011). Efficiently identifying AGNs in all states of accretion and obscuration and accurately understanding their properties and structure is a key step to understand how galaxies evolve with cosmic time.

AGNs are among the most luminous objects in the universe. Most of the radiated energy is thermally generated by the accretion disk surrounding the central supermassive black hole (SMBH) within scales of $\sim 1 \mathrm{AU}$, with a spectrum that is well

\footnotetext{
${ }^{14}$ NASA Postdoctoral Program Fellow.
}

approximated by a declining power law shortward of soft X-ray wavelengths (e.g., Shakura \& Sunyaev 1973). A considerable fraction of this luminosity is absorbed by dust surrounding the SMBH on scales of $\sim 1 \mathrm{pc}$, which is heated up to temperatures reaching its sublimation limit $(\sim 1500 \mathrm{~K})$ and re-radiates it in the IR. The dust distribution is typically thought to have a quasitoroidal shape (see, e.g., Urry \& Padovani 1995), with a scale height and opening angle that depends on the luminosity of the accretion disk (see, e.g., Simpson 2005). The dust emission dominates the mid-IR emission in an AGN and rises as a power law toward longer wavelengths, providing AGNs with their characteristic red mid-IR colors (e.g., Elvis et al. 1994; Stern et al. 2005; Richards et al. 2006; Assef et al. 2010), which allows their identification even when the accretion disk emission is blocked by the dust torus, as it differs strongly from the Rayleigh-Jeans emission of the stellar population that dominates the mid-IR spectrum of inactive galaxies.

The Wide-field Infrared Survey Explorer (WISE; Wright et al. 2010 ) is a NASA satellite with a $40 \mathrm{~cm}$ aperture that imaged 
the whole sky in four mid-IR bands, centered at 3.4, 4.6, 12, and $22 \mu \mathrm{m}$. We refer to these bands as $\mathrm{W} 1, \mathrm{~W} 2, \mathrm{~W} 3$, and $\mathrm{W} 4$, respectively. The fully cryogenic WISE science mission started in 2010 January and ended in August of the same year; all of these data have been publicly available since 2012 March. While not one of its main goals, WISE is well suited to studying AGNs as its bands are sensitive to their characteristic warm dust emission and are little affected by the obscuration expected from either the dust in the torus or in the interstellar medium (ISM) of the host galaxy. In a companion work, Stern et al. (2012, Paper I) investigated the power of WISE to identify AGNs based solely on the W1-W2 mid-IR color by comparing to known AGNs in the COSMOS field. The selection also necessarily entails a flux cut, which is relatively shallow given the low ecliptic latitude of the COSMOS field and thus lower WISE coverage depth. Using a set of AGNs in the field selected from the Spitzer Infrared Array Camera (IRAC; Fazio et al. 2004) colors according to the criteria developed by Stern et al. (2005) as a control sample, we determined that at the depth of the COSMOS field a very simple selection criterion of $\mathrm{W} 1-\mathrm{W} 2 \geqslant 0.8$ and $\mathrm{W} 2<15.05$ produced an AGN sample with a contamination of only $5 \%$ and recovered nearly $80 \%$ of the IRAC-selected AGNs in the field to that WISE depth. The $61.9 \pm 5.4 \mathrm{deg}^{-2}$ space density of these AGNs is about three times higher than that of similar bolometric luminosity Type 1 AGNs found at optical wavelengths by the Sloan Digital Sky Survey (SDSS; Richards et al. 2002). The color of this criterion is similar to that proposed earlier by Assef et al. (2010, W1-W2 > 0.85) using mock WISE data constructed from spectral energy distribution (SED) models calibrated in this wavelength range by Spitzer data. Assef et al. (2010) showed that WISE and SDSS are sensitive to AGNs of the same bolometric luminosities for $z<4$, implying that the increased census is due to the sensitivity of WISE to those objects obscured by dust. In fact, Paper I showed that the distribution of the X-ray hardness ratios of the WISE-selected AGNs is, as expected, consistent with a considerable number of dust-obscured AGNs.

Because of the polar orbit and near continuous observing strategy of WISE, the depth of a field depends strongly on ecliptic latitude. The $2 \mathrm{deg}^{2}$ COSMOS field, close to the ecliptic, is representative of the shallowest WISE fields. In this work we extend the work presented in Paper I to the much larger, $9 \mathrm{deg}^{2}$ NOAO Deep Wide-Field Survey (NDWFS; Jannuzi \& Dey 1999) Boötes field, which has considerably deeper WISE observations due to its higher ecliptic latitude. In Section 2, we describe the data sets and SED-fitting models which we use to extend the AGN selection criteria of Paper I to fainter WISE fluxes in Section 3. In Section 4, we study the broadband SEDs of the WISE AGN candidates and assess the accuracy with which we can estimate photometric redshifts for them. Finally, in Section 5, we study the distribution of the obscuring dust in AGNs and present a method to correct for sample incompleteness due to reddening. Throughout this work we assume a flat $\Lambda$ CDM cosmology with $H_{0}=73 \mathrm{~km} \mathrm{~s}^{-1}$, $\Omega_{M}=0.3$, and $\Omega_{\Lambda}=0.7$. We refer to all magnitudes in their native photometric system, i.e., AB for ugriz, far-UV (FUV) and near-UV (NUV), and A0 (Vega) for all other bands.

\section{DATA AND MODELING}

\subsection{The NOAO Deep Wide-Field Survey Boötes Field}

NDWFS is a deep imaging survey in $B_{W}, R, I$, and $K$ of two $9 \mathrm{deg}^{2}$ fields in the constellations of Cetus and Boötes. We focus here on the Boötes field, for which follow-up deep imaging has been obtained for a wide range of wavelengths. Boötes also has deep and extensive spectroscopy.

Follow-up imaging of the Boötes field exists from the X-rays with Chandra (XBoötes; Murray et al. 2005) to the radio from the Faint Images of the Radio Sky at Twenty-centimeters (FIRST; Becker et al. 1995) survey, the NRAO VLA Sky Survey (NVSS; Condon et al. 1998), the Westerbork Northern Sky Survey (WENSS; Rengelink et al. 1997), and from de Vries et al. (2002). The whole field was observed with 90 s of exposure per position in the IRAC Shallow Survey (Eisenhardt et al. 2004). The Spitzer Deep, Wide-Field Survey (SDWFS; Ashby et al. 2009) quadrupled this exposure, reaching $5 \sigma$ depths of 19.3, 18.5, 16.3, and 15.6 mag for [3.6], [4.5], [5.8], and [8.0], respectively. Additionally, we also use the Galaxy Evolution Explorer (GALEX; Martin et al. 2005) Deep Imaging Survey (DIS) and All-sky Imaging Survey (AIS) FUV and NUV observations of the field, the $z$-band data of Cool (2007), the near-IR $J, H$, and $K_{s}$ observations of NEWFIRM (Gonzalez et al. 2010), and the MIPS $24 \mu \mathrm{m}$ observations of the MIPS AGN and Galaxy Evolution Survey (MAGES; Jannuzi et al. 2010). For our work, we use 6" aperture magnitudes, corrected for PSF losses, obtained from PSF-matched images in all but the Spitzer bands.

The AGN and Galaxy Evolution Survey (AGES; Kochanek et al. 2012) obtained deep optical spectra of approximately 25,000 sources in the Boötes field with Hectospec (Fabricant et al. 2005) at the Multiple Mirror Telescope (MMT). The survey is designed to be statistically complete for several different samples limited to $I<20$ for galaxy candidates and $I<22.5$ for AGN candidates. AGES is highly complete for AGN candidates to $I<21.5$ (see Kochanek et al. 2012, for details on completeness and selection). The AGN candidates were targeted by their X-ray, radio, and mid-IR properties, but not by their optical colors. This ensures that none of the optical selection biases (see, e.g., Fan 1999) are propagated into the sample. We complement the AGES spectroscopy with 2000 deeper optical spectra from various sources, primarily from Keck (e.g., Eisenhardt et al. 2008). Since these data do not have a uniform selection function, they will only be of limited use in our analysis.

\subsection{WISE Observations}

The WISE mission observed the full sky in four mid-IR photometric bands with an FWHM of $6^{\prime \prime}$ in W1-W3 and 12" in W4. We use the WISE all-sky data release, which includes all observations obtained during the fully cryogenic mission. WISE surveyed the sky in a polar orbit with respect to the ecliptic, simultaneously obtaining images in all four bands. Hence, the number of observations in a field increases with its ecliptic latitude. While fields near the ecliptic were typically observed 12 times, the number can grow to several hundreds near the ecliptic poles (e.g., Jarrett et al. 2011). The median coverage across the sky is approximately 15 frames per passband. In particular, the COSMOS field was observed with a median coverage of 11 frames per passband, well below the median sky coverage. Detailed accounts of the mission are presented by Wright et al. (2010) and in the WISE all-sky data release explanatory supplement. ${ }^{15}$

The NDWFS Boötes field is at an ecliptic latitude of $46 \mathrm{deg}$, and hence WISE obtained an average coverage of 30 frames in each band, reaching $10 \sigma$ depths in $\mathrm{W} 1, \mathrm{~W} 2, \mathrm{~W} 3$, and $\mathrm{W} 4$ of approximately $17.12 \mathrm{mag}, 15.73 \mathrm{mag}, 11.55 \mathrm{mag}$, and $7.83 \mathrm{mag}$.

\footnotetext{
15 http://wise2.ipac.caltech.edu/docs/release/allsky/expsup/
} 
For all sources, we use fluxes obtained through profile fitting. We limit the sample to $\mathrm{S} / \mathrm{N}>3$ in $\mathrm{W} 1$ and $\mathrm{W} 2$, or equivalently to $\mathrm{W} 1<18.50$ and $\mathrm{W} 2<17.11$. We match to other sources in Boötes by finding the closest IRAC [4.5] source within $2^{\prime \prime}$ with the constraint that no WISE (IRAC) source is matched to more than one IRAC (WISE) source. This results in a sample of 111,720 matched sources. We note that the WISE magnitude limit is applied after cross-matching with the IRAC sources.

Detailed comparison between WISE and Spitzer IRAC photometry has shown that WISE profile-fitting fluxes in $\mathrm{W} 1$ and W2 are typically underestimated for faint sources, and that the magnitude of the effect increases with decreasing IRAC flux, reaching offsets of a few tenths of a magnitude for the fainter sources (see section VI.3 of the WISE all-sky data release explanatory supplement for details). No similar effect is observed for W3 and W4. While there is no simple prescription to mitigate it, this bias is unlikely to affect the results of our SED fits, as it is only significant for faint sources for which the deeper IRAC SDWFS magnitudes dominate the $\chi^{2}$ of the fit in the mid-IR. Because of this, we do not attempt to compensate for this WISE calibration issue in our study.

\subsection{Spectral Energy Distribution Modeling}

We rely on SED modeling both to obtain physical insight into our AGN candidates and to obtain photometric redshift $\left(z_{\text {phot }}\right)$ estimates for all objects without available spectroscopic redshifts. To fit the SEDs we use the non-negative basis of low-resolution, UV through mid-IR SED templates for AGNs and galaxies of Assef et al. (2010). The basis consists of four empirically derived SED templates, where every object is modeled as a non-negative combination of the three galaxy SED templates (roughly corresponding to E, Sbc, and Im types) and the single AGN template. For the AGN template alone, we allow reddening with a strength parameterized by $E(B-V)$. For high-redshift sources, we model the IGM absorption following Fan et al. (2006) for $\operatorname{Ly} \alpha$ and $\operatorname{Ly} \beta$ absorption and StenglerLarrea et al. (1995) for Lyman limit systems. The strength of the IGM absorption can also be fit beyond the standard mean absorption law, although this extra degree of freedom often has a negative impact on the accuracy of photometric redshifts. A weak prior is used to keep $E(B-V)$ as small as possible with the secondary effect that obscuration values may be slightly underestimated in some cases. This prior is required to avoid an extremely reddened AGN component being used to improve the fit to the longest wavelength bands (primarily W3, W4, and MIPS $24 \mu \mathrm{m}$ ) in an inactive galaxy without affecting the SED at shorter wavelengths. Also, this prior can lower the possible degeneracy between a red stellar spectrum and a reddened AGN in $z \gtrsim 1$ inactive galaxies with little or no rest-frame mid-IR constraints, although we note this is very unlikely to happen in our W2-selected sample given the deep SDWFS IRAC [5.8] and [8.0] observations. We refer the reader to Assef et al. (2010) for further details on the $E(B-V)$ prior.

We follow the prescription detailed in Assef et al. (2010) to obtain photometric redshifts and fit the SEDs. Since photometric redshifts using $24 \mu \mathrm{m}$ photometry have lower accuracies when using these templates (see Assef et al. 2010, for details), we derive photometric redshifts for all objects in the sample using only the broadband photometry from FUV to W3. Adding MIPS $24 \mu \mathrm{m}$ and $\mathrm{W} 4$ photometry, however, does not qualitatively alter our results. We assume the standard mean IGM absorption and use a luminosity prior for the galaxy components based on the Las Campanas Redshift Survey $r$-band luminosity function
(Lin et al. 1996). We discuss the precision of the photometric redshifts in Section 4.1.

After obtaining photometric redshift estimates, we re-fit the SEDs of all objects now including the W4 and MIPS $24 \mu \mathrm{m}$ channels, and also fit for the strength of the IGM absorption. Whenever possible, we use spectroscopic redshifts $\left(z_{\text {spec }}\right)$. This approach ensures that we get the best SED model possible for each object. Several authors have determined that photometric redshifts for Type 1 AGNs obtained solely with broadband filter photometry can be wildly inaccurate (see, e.g., RowanRobinson et al. 2008; Salvato et al. 2009; Assef et al. 2010); this is discussed further in Section 4.1 in the context of our study. However, our spectroscopic data are particularly deep and complete for AGNs (see Section 2.1), somewhat mitigating this issue.

In order to reliably separate AGNs from inactive galaxies, we use the parameter

$$
\hat{a} \equiv \frac{L_{\mathrm{AGN}}}{L_{\mathrm{host}}+L_{\mathrm{AGN}}},
$$

where the luminosities correspond to the integrated specific luminosities of the best-fit templates over the $0.1-30 \mu \mathrm{m}$ wavelength range for the AGN template and 0.03-30 $\mu \mathrm{m}$ for the host galaxy templates (see Assef et al. 2010, for details). The specific luminosities are calculated after correcting the AGN component for the best-fit value of the reddening. We refer to these as bolometric luminosities for the rest of the paper. Note that Assef et al. (2010) determined that $\hat{a}$ is insensitive to photometric redshift uncertainties as long as enough data exist to constrain the fit, in the sense that $\hat{a}$ can still be accurately determined for objects where reliable photometric redshifts cannot be measured. That is to say, while it is challenging to measure accurate photometric redshifts for AGNs, particularly for Type 1 AGNs, we are able to accurately disentangle the relative fractions of starlight and nuclear emission even when the redshift estimate is significantly in error. A general characterization of this accuracy beyond that in Assef et al. (2010) is presented in Appendix A.

\section{WISE AGN COLOR SELECTION}

In this section, we study the completeness and reliability of WISE AGN selection. First, we discuss the criterion of Paper I applied to the deeper WISE data in the Boötes field, while in Section 3.2 we improve our method by also considering the observed W2 magnitude of the sources. In Section 3.3, we compare our new method with others in the literature.

\subsection{Magnitude-independent AGN Color Selection}

In Paper I, we investigated the distribution of quasars in WISE color space in the COSMOS survey field. We found that for objects with $\mathrm{W} 2<15.05 \mathrm{mag}$ (W2 $\mathrm{S} / \mathrm{N} \geqslant 10$ at that ecliptic latitude), the simple color cut based on the two shortest wavelength WISE bands,

$$
\mathrm{W} 1-\mathrm{W} 2 \geqslant 0.8
$$

provides an effective criterion to separate AGNs from inactive galaxies. When compared to the IRAC color selection method of Stern et al. (2005; see Assef et al. 2010 for discussion about its reliability), the WISE color criterion selection recovers $78 \%$ of the IRAC-selected AGNs with a 95\% reliability. Notably, six of the AGN candidates selected by IRAC and WISE were not detected in the $1.8 \mathrm{Ms}$ Chandra survey of the COSMOS 


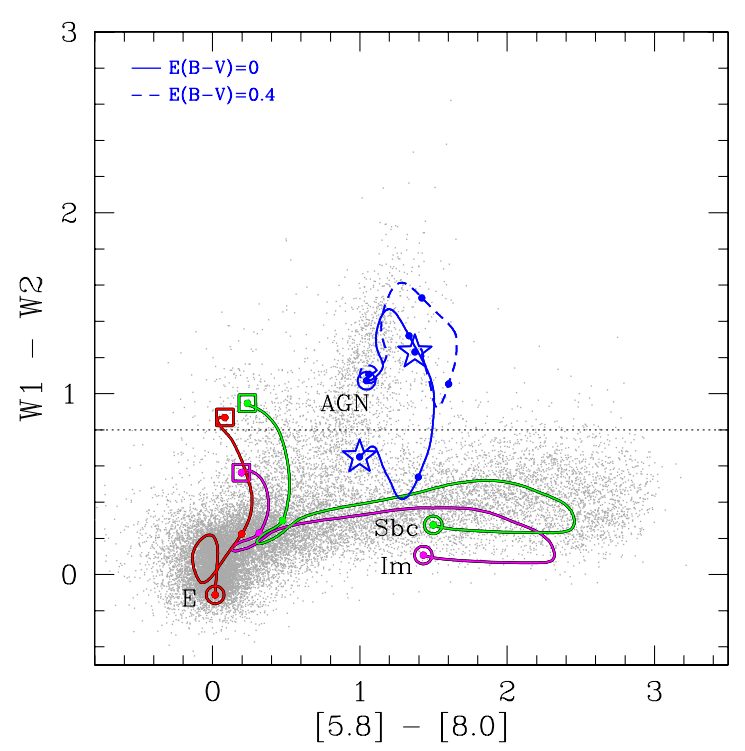

Figure 1. Distribution of [5.8]-[8.0] and W1-W2 colors in the Boötes field. The lines show the colors of the galaxy and AGN SED templates of Assef et al. (2010). The colors of the galaxy templates E (red line), Sbc (green line), and Im (magenta line) are shown between redshifts 0 (open circle) and 2 (open square), with dots in the tracks in steps of $\Delta z=1$. The AGN template is shown without reddening (solid blue line) and with $E(B-V)=0.4$ (dashed blue line), in the redshift interval between $z=0$ (open circle) and $z=6$ (open star). Dots in the AGN color tracks are spaced by $\Delta z=2$. The gray dots show all the WISE sources in the NDWFS field with W2 $<15.73$.

(A color version of this figure is available in the online journal.)

field (C-COSMOS; Elvis et al. 2009), suggesting they may be Compton-thick (see Paper I for details). The reason behind the success of this criterion is clearly illustrated in Figure 1. This figure shows mid-IR color as a function of redshift for the AGN and galaxy SED templates of Assef et al. (2010). For $z \lesssim 3$, the W1-W2 color of unobscured AGNs is well above the color cut of $0.8 \mathrm{mag}$. At higher redshift, reddened AGNs $(E(B-V) \gtrsim 0.4)$ can also be redder than this color cut. In practice, however, it is exceedingly uncommon to find high-redshift, highly reddened AGNs bright enough to be detected by WISE and so much more luminous that their host galaxy dominates the rest-frame optical emission. Indeed, in Paper I we found no quasars with $z>3$ in the sample. We also find very few galaxies with $z \gtrsim 1$, since the WISE observations of the COSMOS field are not deep enough to find normal galaxies at high redshifts. While other populations such as some ULIRGs and brown dwarfs can have even redder $\mathrm{W} 1-\mathrm{W} 2$ colors, they are too rare in comparison to AGNs to be a significant source of contamination. Note that Figure 1 implies that this W1-W2 color selection is biased against AGNs which are faint with respect to their host galaxies. If the flux in the WISE bands is dominated by the galaxy, the colors will drop below the selection limit, moving toward the galaxy locus at $\mathrm{W} 1-\mathrm{W} 2 \sim 0$. We discuss this further in Section 4.3.

The criteria of Paper I are readily applicable to the all-sky WISE survey and are demonstrated to be both reliable and complete to the shallow depth of the WISE observations of the COSMOS field. However, due to the limited size of that field, it does not have the statistical power to address many interesting and pressing issues in AGN studies, such as AGN evolution, accretion rates, and dust distributions. More importantly, since most of the WISE survey area has deeper coverage than in the COSMOS field, alternative selection criteria are valuable for a census of WISE-selected AGNs in these deeper regions. Jarrett et al. (2011) have shown that in the deepest WISE fields at

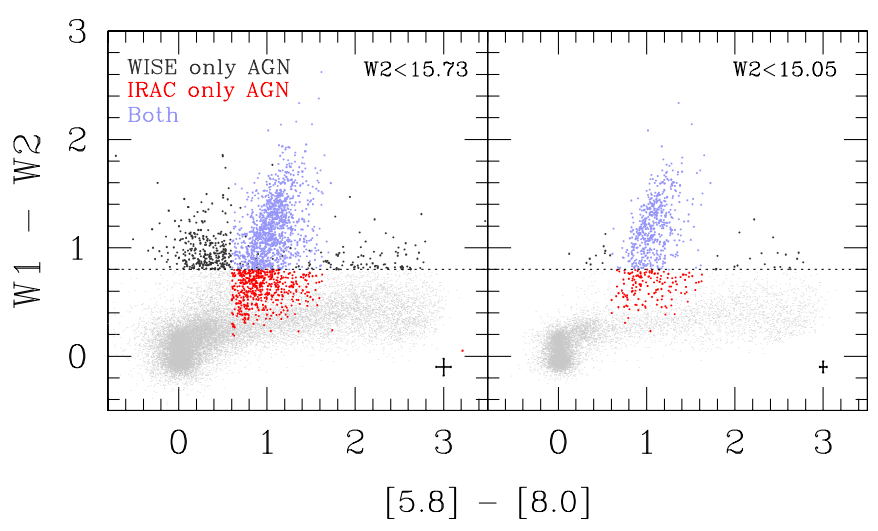

Figure 2. WISE W1-W2 vs. SDWFS [5.8]-[8.0] colors for WISE sources in the NDWFS Boötes field. The left panel shows sources with W2 $<15.73$, the $10 \sigma$ WISE detection limit in the Boötes field, while the right panel shows sources limited to $\mathrm{W} 2<15.05$, corresponding to the $\mathrm{W} 2 \mathrm{~S} / \mathrm{N}>10 \mathrm{limit}$ in the COSMOS field. Objects are separated into non-AGN candidates (light gray dots), WISE and IRAC AGN candidates (blue dots), WISE-only candidates (black dots), and IRAC-only candidates (red dots). The median photometric uncertainty for each sample is shown in the lower right corner of each panel.

(A color version of this figure is available in the online journal.)

the ecliptic poles, where $\mathrm{W} 1$ and $\mathrm{W} 2$ are confusion limited, the addition of W3 is a very useful aid in the identification of AGNs. Our intention is to bridge these two extremes, proposing a robust WISE AGN selection technique for fields with intermediate depth.

In order to extend the study of Paper I, we turn to the NDWFS Boötes field, which helps with both issues highlighted above: it has a WISE median coverage of 30 frames, almost three times that of COSMOS, and extends over $9 \mathrm{deg}^{2}$, an area 4.5 times larger. We start by replicating the selection criterion of Paper I in the Boötes field, but up to the W2 $10 \sigma$ depth provided by the full co-added data. While this implies a sample 0.68 mag deeper, it maintains the error properties of the sample and so provides a meaningful comparison.

The left panel of Figure 2 shows the WISE color distribution of sources in our sample. Comparing to sources selected as AGNs by their IRAC colors (Stern et al. 2005), we find that a simple $\mathrm{W} 1-\mathrm{W} 2 \geqslant 0.8$ color cut identifies $70 \%$ of the IRACselected AGNs with $70 \%$ reliability. Compared to applying this cut at the $10 \sigma$ WISE depth of COSMOS as reported in Paper I, the drop in completeness is relatively small, from $78 \%$ to $70 \%$. The decrease in reliability from $95 \%$ to $70 \%$ is very significant, however, and is simply due to the modest increase in field depth. If we limit the Boötes field analysis to the $10 \sigma \mathrm{W} 2$ level of the COSMOS field (W2 $<15.05$, right panel of Figure 2), we recover similar AGN demographics to that reported in Paper I, with $78 \%$ completeness and $94 \%$ reliability.

The lower completeness means an increase in IRAC-selected AGNs detected but not identified by the simple WISE color criterion. This is likely due to a combination of (1) a small number of $z \gtrsim 3$ Type 1 AGNs, which are known to be excluded by the Paper I color selection; (2) low-redshift, lowluminosity AGNs with hosts bright enough to move their midIR colors below the WISE selection limit, but red enough to be picked by the Stern et al. (2005) IRAC selection criteria; and (3) a higher incidence of contamination by $z \sim 0.5$ star-forming galaxies to the IRAC selection criterion, which artificially lowers the completeness-though we note that this contamination is expected to be small at the depth of SDWFS (see Assef et al. 2010; Donley et al. 2012). 


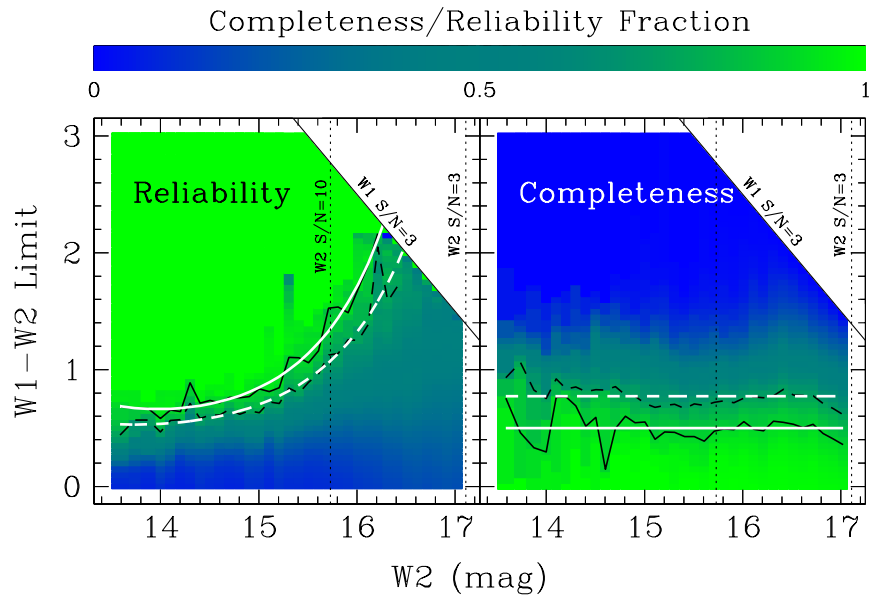

Figure 3. Reliability (left panel) and completeness (right panel) of AGN candidates defined by $\hat{a}>0.5$ selected by a color cut on W1 $-\mathrm{W} 2$ as a function of W2 magnitude. Reliability and completeness of $90 \%(75 \%)$ are shown as a function of magnitude by the solid (dashed) black lines. Objects redder than the top right corner of the panels are missing due to the $\mathrm{W} 1 \mathrm{~S} / \mathrm{N}>3$ requirement. The proposed reliability-optimized criteria (Equation (3)) for $90 \%\left(R_{90}\right)$ and $75 \%\left(R_{75}\right)$ reliability are shown in the left panel by the white solid and dashed lines, respectively. The completeness-optimized criteria (Equation (4)) for $90 \%$ $\left(C_{90}\right)$ and $75 \%\left(C_{75}\right)$ completeness are shown in the right panel with the same respective line styles.

(A color version of this figure is available in the online journal.)

The cause of the significantly lower reliability obtained in the Boötes field compared to the COSMOS field is readily apparent in Figure 2. The modestly deeper WISE sample increases the number of contaminating galaxies, particularly to the left of the QSO locus. These correspond to high-redshift $(z \sim 1-1.5)$ galaxies. The observed W2 magnitude of $z \sim 1-1.5 L_{*}$ galaxies evolves very slowly with redshift (see, e.g., Figure 1 of Eisenhardt et al. 2008), so there is a huge increase in contamination as soon as the W1 magnitude limit is deep enough to begin including these galaxies. In the Stern et al. (2005) IRAC selection criterion this problem is controlled using the [5.8]-[8.0] color, but the longer wavelength WISE bands are too shallow to help.

\subsection{Magnitude-dependent AGN Color Selection}

It is apparent from Figure 2 that an improved method to select AGNs may be possible if we allow our color cut to evolve with magnitude since the major contaminants are either lowredshift, nearby star-forming galaxies which are intrinsically faint or high-redshift, passive galaxies that are luminous enough to be bright in the WISE bands. In order to design a magnitudedependent AGN color selection method that is applicable over the whole sky, we will go to fainter WISE fluxes than afforded by the $10 \sigma$ W2 limit in the Boötes field. Note, however, that as we go to fainter W2 magnitudes, it becomes unreliable to use an AGN control sample based on the IRAC color criteria of Stern et al. (2005), as it is susceptible to contamination by high-redshift galaxies once the errors in the SDWFS IRAC [5.8] and [8.0] fluxes become too large (see, e.g., Donley et al. 2012). Instead, we define the control sample as all objects whose best-fit UV-mid-IR SEDs have a strong AGN component, as indicated by requiring $\hat{a}>0.5$. For significantly lower levels of AGN activity, it becomes necessary to differentiate between objects where the AGN component of the fit is real and when it has only been used to mathematically improve the $\chi^{2}$ to accommodate lower quality photometry or mimic a galaxy component missing

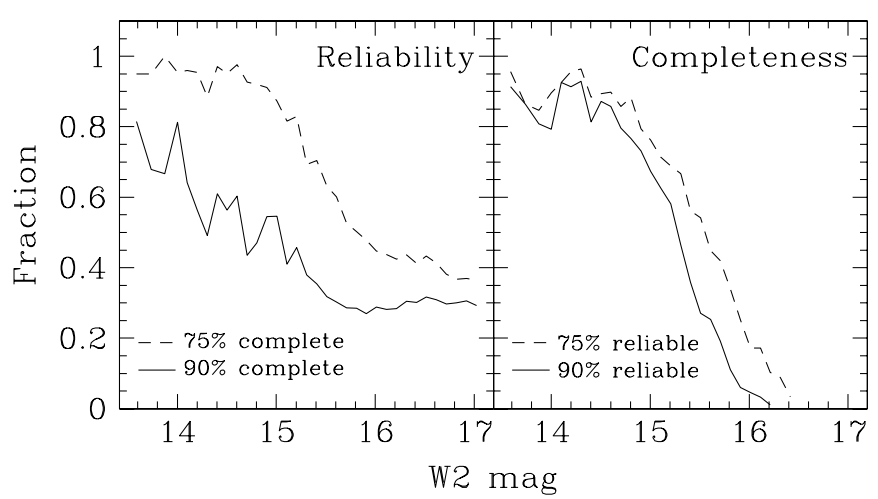

Figure 4. Left panel shows the reliability as a function of W2 magnitude for the $90 \%$ (solid line) and $75 \%$ (dashed line) completeness-optimized AGN selection criteria. The right panel shows the completeness as a function of W2 magnitude for the $90 \%$ (solid line) and $75 \%$ (dashed line) reliability-optimized AGN selection criteria.

from the templates. This falls beyond the scope of the current work, and a full analysis on this topic is presented by S. M. Chung et al. (in preparation). Assef et al. (2010) have shown that the Stern et al. (2005) criterion is biased toward objects with large $\hat{a}$ values (see also Section 4.3), so we are not considerably changing the physical properties of the control sample by using this definition.

Figure 3 shows the completeness and reliability obtained as a function of W2 magnitude and the minimum W1-W2 color limit adopted to select AGNs. We have required a minimum detection threshold of $3 \sigma$ for $\mathrm{W} 1$ in order to have a reasonably precise WISE color. At bright W2, a color cut of 0.6 is sufficient to obtain high reliability and high completeness. Toward fainter W2 magnitudes, high reliability requires redder color cuts in order to remove contaminating galaxies, which also leads to lower completeness. The completeness of a color cut is relatively independent of W2 magnitude.

Figure 3 shows the bluest W1-W2 color at which $90 \%$ and $75 \%$ reliability is reached for a given W2 magnitude. While there is significant noise in these curves, they are reasonably well described by an exponential in $\mathrm{W}^{2}$. Hence, we propose a WISE AGN color selection limit optimized for reliability given by

$$
\mathrm{W} 1-\mathrm{W} 2>\alpha_{\mathrm{R}} \exp \left\{\beta_{\mathrm{R}}\left(\mathrm{W} 2-\gamma_{\mathrm{R}}\right)^{2}\right\}
$$

For $\mathrm{W} 2<17.11$, we achieve a reliability of $\sim 90 \%$ with $\left(\alpha_{R 90}, \beta_{R 90}, \gamma_{R 90}\right)=(0.662,0.232,13.97)$. The corresponding values for a reliability of $\sim 75 \%$ are $\left(\alpha_{R 75}, \beta_{R 75}, \gamma_{R 75}\right)=$ $(0.530,0.183,13.76)$. The $90 \% \quad(75 \%)$ reliability criterion reaches our imposed $\mathrm{W} 1 \mathrm{~S} / \mathrm{N}>3$ limit at a $\mathrm{W} 2$ magnitude of 16.26 (16.45). The right panel of Figure 4 shows the completeness as a function of W2 magnitude for each of the criteria. Only considering objects brighter than this limit in W2, the $90 \%$ reliability criterion identifies 1174 AGN candidates, of which $1060(90 \%)$ have their bolometric luminosities dominated by the AGN (e.g., $\hat{a}>0.5$ ). For the $75 \%$ reliability criterion, we identify 2306 AGN candidates, of which $1752(76 \%)$ are AGN dominated. At a shallower depth of $\mathrm{W} 2<15.73$, corresponding to $\mathrm{S} / \mathrm{N}>10$ in the Boötes field, the $90 \%$ reliability curve identifies 1051 AGN candidates, of which 950 (90\%) are AGN dominated, while the $75 \%$ reliability curve identifies 1582 AGN candidates, of which 1200 (76\%) are AGN dominated. For comparison, a simple W1-W2 $\geqslant 0.8$ cut (e.g., similar to the criterion of Paper I, but without its magnitude cut) finds 1746 AGN candidates with $74 \%$ reliability at a depth of $\mathrm{W} 2=15.73$. This census 
Table 1

Surface Density of AGN Candidates at W2 $<17.11^{\mathrm{a}}$

\begin{tabular}{|c|c|c|c|c|c|}
\hline Selection Criterion & $\begin{array}{c}N \\
\left(\operatorname{deg}^{-2}\right)\end{array}$ & $\begin{array}{c}N(\hat{a}>0.5) \\
\left(\operatorname{deg}^{-2}\right)\end{array}$ & $\begin{array}{c}\text { Reliability } \\
\text { Fraction }\end{array}$ & $\begin{array}{c}\text { Completeness } \\
\text { Fraction }\end{array}$ & $\begin{array}{c}\text { Bands } \\
\text { Used }\end{array}$ \\
\hline \multicolumn{6}{|c|}{ WISE AGN selection } \\
\hline$R_{90}$ & 130 & 118 & 0.90 & 0.09 & $\mathrm{~W} 1, \mathrm{~W} 2$ \\
\hline$R_{75}$ & 256 & 195 & 0.76 & 0.17 & $\mathrm{~W} 1, \mathrm{~W} 2$ \\
\hline$C_{90}$ & 3702 & 1152 & 0.31 & 0.89 & $\mathrm{~W} 1, \mathrm{~W} 2$ \\
\hline$C_{75}$ & 2117 & 929 & 0.44 & 0.72 & $\mathrm{~W} 1, \mathrm{~W} 2$ \\
\hline $\mathrm{W} 1-\mathrm{W} 2 \geqslant 0.8^{\mathrm{b}}$ & 2000 & 901 & 0.45 & 0.70 & $\mathrm{~W} 1, \mathrm{~W} 2$ \\
\hline Jarrett et al. (2011) & 469 & 268 & 0.57 & 0.21 & $\mathrm{~W} 1, \mathrm{~W} 2, \mathrm{~W} 3^{\mathrm{c}}$ \\
\hline Mateos et al. (2012) & 391 & 256 & 0.65 & 0.20 & $\mathrm{~W} 1, \mathrm{~W} 2, \mathrm{~W} 3$ \\
\hline Mateos et al. (2012) & 83 & 68 & 0.82 & 0.05 & $\mathrm{~W} 1, \mathrm{~W} 2, \mathrm{~W} 3, \mathrm{~W} 4$ \\
\hline Assef et al. (2010) & 1785 & 841 & 0.47 & 0.65 & $\mathrm{~W} 1, \mathrm{~W} 2$ \\
\hline Assef et al. (2010) & 44 & 43 & 0.97 & 0.03 & $\mathrm{~W} 1, \mathrm{~W} 2, \mathrm{~W} 3, \mathrm{~W} 4$ \\
\hline Wu et al. (2012b) & 3218 & 1109 & 0.34 & 0.86 & $\mathrm{~W} 1, \mathrm{~W} 2$ \\
\hline \multicolumn{6}{|c|}{ Other infrared AGN selection } \\
\hline Messias et al. (2012) & 662 & 543 & 0.82 & 0.42 & $K_{s},[4.5],[8.0]$ \\
\hline Messias et al. (2012) & 314 & 280 & 0.89 & 0.22 & $K_{s},[4.5],[8.0]$, MIPS $24 \mu \mathrm{m}$ \\
\hline Stern et al. (2005) & 986 & 659 & 0.67 & 0.51 & {$[3.6],[4.5],[5.8],[8.0]$} \\
\hline Lacy et al. (2004) & 2888 & 1029 & 0.36 & 0.79 & {$[3.6],[4.5],[5.8],[8.0]$} \\
\hline Lacy et al. (2007) & 1297 & 735 & 0.57 & 0.57 & {$[3.6],[4.5],[5.8],[8.0]$} \\
\hline
\end{tabular}

Notes. WISE AGN selection criteria $R_{90}, R_{75}, C_{90}$, and $C_{75}$ are described by Equations (3) and (4). The remaining WISE AGN selection criteria are as follows. Jarrett et al. (2011): W2-W3 > 2.2, W2-W3 > 4.2, W1-W2 > 0.1 (W2-W3)+0.38, W1-W2 $<1.7$, and object is not a star; Mateos et al. (2012) three-band: W2-W3 $>2.157$, $\mathrm{W} 1-\mathrm{W} 2>0.315$ (W2-W3)-0.222, W1-W2 < 0.315(W2-W3)+0.796; Mateos et al. (2012) four-band: W3-W4 $\geqslant$ 1.76, W1-W2 > 0.50(W3-W4)-0.405, W1-W2 < 0.50(W3-W4)+0.979; Assef et al. (2010) two-band: W1-W2 > 0.85; Assef et al. (2010) four-band: W3-W4 > 2.1, W1-W2 > 0.85, W1-W2 > 1.67(W3-W4)-3.41; Wu et al. (2012b): W1-W2 > 0.57. For the other infrared AGN selection criteria we refer the reader to the original studies. a The effective W2 limits for the $R_{90}$ and $R_{75}$ criteria are 16.26 and $16.45 \mathrm{mag}$, respectively, due to the W1 $\mathrm{S} / \mathrm{N}>3$ requirement of our sample.

$\mathrm{b}$ This criterion corresponds to the color cut proposed by Paper I without the magnitude limit W2 $<15.05$.

${ }^{\mathrm{c}} \mathrm{W} 4$ is also used if detected.

increases to 17,997 AGN candidates to a depth of $\mathrm{W} 2=17.11$, albeit with a reliability that drops to $45 \%$. These statistics are summarized in Tables 1 and 2. In the next section (Section 3.3) we compare this new magnitude-dependent AGN selection criterion to several other WISE AGN selection criteria that have recently been proposed in the literature.

Figure 3 also shows the reddest W1-W2 color at which $90 \%$ and $75 \%$ completeness is reached for objects with bolometric luminosities dominated by the AGN emission. In this case, a reasonable description of the completeness boundary is given by the magnitude independent color cut

$$
\mathrm{W} 1-\mathrm{W} 2>\delta_{\mathrm{C}},
$$

where $\delta_{C 90}=0.50$ for $90 \%$ completeness and $\delta_{C 75}=0.77$ for $75 \%$ completeness. Note that the $75 \%$ completeness criterion is basically equivalent to the cut proposed by Paper I, shown in Equation (2), but without the flux cut. The left panel of Figure 4 shows the reliability as a function of W2 magnitude for each of the criteria. It is important to stress that these criteria are appropriate only for strong AGNs with respect to their hosts due to the $\hat{a}>0.5$ requirement.

While the magnitude dependence in the reliability-optimized criterion is caused in part by the much higher number of highredshift galaxies at fainter fluxes (see discussion in Section 3.1), it is also driven by the increasingly large errors in W1 and W2 at lower signal-to-noise ratio $(\mathrm{S} / \mathrm{N})$. Since the $\mathrm{S} / \mathrm{N}$ of WISE observations varies significantly across the sky, in principle, $\alpha_{R}$, $\beta_{R}$, and $\gamma_{R}$ may depend on ecliptic latitude. To test the strength of this dependence, we simulate the distribution of W1-W2 and
W2 magnitudes for different WISE field depths and estimate $\alpha_{R}$, $\beta_{R}$, and $\gamma_{R}$ in each case. For this we use the magnitudes obtained from the SED modeling of every object and we approximate that the $\mathrm{S} / \mathrm{N}$ for a flux $F$ depends on field depth as

$$
\frac{\mathrm{S}}{\mathrm{N}}=K_{1} \sqrt{\frac{N_{F}}{30}} \frac{F}{\sqrt{F+F_{\text {Sky }}}},
$$

where $N_{F}$ is the number of individual WISE $11 \mathrm{~s}$ frames used to build the catalog image, $K_{1}$ is a constant, and $F_{\text {Sky }}$ is the background flux. This formulation neglects the effects of confusion as well as the variation of $F_{\text {Sky }}$ with sky position, which is not uniform across the sky. However, our approximation should give a good general idea of how the parameters in question vary with $N_{F}$. As discussed earlier, $N_{F}^{\text {Bootes }}=30$, and we use the Boötes data to fit for $K_{1}$ and $F_{\text {Sky }}$. We find that from $N_{F}=10$ to 50, no significant variation is observed for the $\alpha_{R}$ and $\gamma_{R}$ parameters for both the $90 \%$ and $75 \%$ criteria. The parameter $\beta_{R}$ is observed to decrease linearly by a factor of $\sim 4$ between $N_{F}=8$ and $N_{F}=25$ and is approximately independent of the depth of the field for $N_{F} \gtrsim 25$. Considering then a modified $\beta_{R}^{\prime}=\beta_{R}^{\text {Boo }}\left(5.41-0.176 N_{F}\right)$ may be necessary to achieve the proposed reliability levels in WISE fields with $N_{F}<25$, where $\beta_{R}^{\text {Boo }}$ is the value obtained for the Boötes field. We also repeat the experiment for the completeness optimized criteria and find that in the same range of $N_{F}, \delta_{C}$ is approximately constant.

We refer to the reliability-optimized selection as $R_{90}$ and $R_{75}$ for $90 \%$ and $75 \%$ reliability, respectively, while $C_{90}$ and $C_{75}$ refer to the completeness-optimized criteria. Whether 
Table 2

Surface Density of AGN Candidates at W2 $<15.73$

\begin{tabular}{|c|c|c|c|c|c|}
\hline Selection Criterion & $\begin{array}{c}N \\
\left(\operatorname{deg}^{-2}\right)\end{array}$ & $\begin{array}{c}N(\hat{a}>0.5) \\
\left(\operatorname{deg}^{-2}\right)\end{array}$ & $\begin{array}{c}\text { Reliability } \\
\text { Fraction }\end{array}$ & $\begin{array}{l}\text { Completeness } \\
\text { Fraction }\end{array}$ & $\begin{array}{l}\text { Bands } \\
\text { Used }\end{array}$ \\
\hline \multicolumn{6}{|c|}{ WISE AGN selection } \\
\hline$R_{90}$ & 117 & 106 & 0.90 & 0.53 & $\mathrm{~W} 1, \mathrm{~W} 2$ \\
\hline$R_{75}$ & 176 & 133 & 0.76 & 0.67 & $\mathrm{~W} 1, \mathrm{~W} 2$ \\
\hline$C_{90}$ & 439 & 177 & 0.40 & 0.88 & $\mathrm{~W} 1, \mathrm{~W} 2$ \\
\hline$C_{75}$ & 194 & 144 & 0.74 & 0.72 & $\mathrm{~W} 1, \mathrm{~W} 2$ \\
\hline $\mathrm{W} 1-\mathrm{W} 2 \geqslant 0.8^{\mathrm{a}}$ & 182 & 139 & 0.77 & 0.69 & $\mathrm{~W} 1, \mathrm{~W} 2$ \\
\hline Jarrett et al. (2011) & 166 & 128 & 0.77 & 0.64 & $\mathrm{~W} 1, \mathrm{~W} 2, \mathrm{~W} 3^{\mathrm{b}}$ \\
\hline Mateos et al. (2012) & 161 & 129 & 0.80 & 0.64 & $\mathrm{~W} 1, \mathrm{~W} 2, \mathrm{~W} 3$ \\
\hline Mateos et al. (2012) & 69 & 56 & 0.80 & 0.28 & $\mathrm{~W} 1, \mathrm{~W} 2, \mathrm{~W} 3, \mathrm{~W} 4$ \\
\hline Assef et al. (2010) & 161 & 130 & 0.81 & 0.65 & $\mathrm{~W} 1, \mathrm{~W} 2$ \\
\hline Assef et al. (2010) & 39 & 38 & 0.98 & 0.19 & $\mathrm{~W} 1, \mathrm{~W} 2, \mathrm{~W} 3, \mathrm{~W} 4$ \\
\hline Wu et al. (2012b) & 347 & 170 & 0.49 & 0.85 & $\mathrm{~W} 1, \mathrm{~W} 2$ \\
\hline \multicolumn{6}{|c|}{ Other infrared AGN selection } \\
\hline Messias et al. (2012) & 166 & 152 & 0.91 & 0.76 & $K_{s},[4.5],[8.0]$ \\
\hline Messias et al. (2012) & 107 & 102 & 0.96 & 0.51 & $K_{s},[4.5],[8.0]$, MIPS $24 \mu \mathrm{m}$ \\
\hline Stern et al. (2005) & 194 & 157 & 0.81 & 0.79 & {$[3.6],[4.5],[5.8],[8.0]$} \\
\hline Lacy et al. (2004) & 484 & 183 & 0.38 & 0.92 & {$[3.6],[4.5],[5.8],[8.0]$} \\
\hline Lacy et al. (2007) & 262 & 172 & 0.66 & 0.86 & {$[3.6],[4.5],[5.8],[8.0]$} \\
\hline
\end{tabular}

Notes.

a This criterion corresponds to the color cut proposed by Paper I without the magnitude limit W2 $<15.05$.

b W4 is also used if detected.

scientific interest lies in maximizing completeness or reliability depends on the problem at hand. However, it will be most common to wish to maximize reliability, so in the next sections we will focus on results for the highest reliability selection.

\subsection{Comparison with the Literature}

AGN identification using mid-IR broadband photometry is now a well-studied problem. The first classification schemes were developed for Spitzer IRAC and MIPS photometry (see, e.g., Lacy et al. 2004, 2007; Stern et al. 2005; Alonso-Herrero et al. 2006; Messias et al. 2012) and have been shown to be very successful in terms of both reliability and completeness. In the previous section we developed four AGN identification schemes using WISE W1 and W2 photometry, optimized to produce samples with different levels of either reliability or completeness. Several other WISE criteria have also recently been developed. Here we briefly discuss several of these criteria and discuss how they compare to our selection criteria. This is meant to be an illustrative rather than an exhaustive exercise; we do not discuss all the published mid-IR selection techniques.

Tables 1, 2, and 3 show the surface density of AGN candidates, their reliability, and their completeness for the selection criteria of Section 3.2, Paper I, Jarrett et al. (2011), Mateos et al. (2012), and $\mathrm{Wu}$ et al. (2012b). Table 1 restricts the samples to W2 < 17.11 (W2 S/N > 3 in Boötes), while Table 2 uses the more restrictive flux cut of $\mathrm{W} 2<15.73$ (W2 S/N $>10$ in Boötes). The samples used in all tables are also restricted to $\mathrm{W} 1<18.50$, but no restriction is applied in W3 and W4. For completeness, Table 3 further limits the sample to W2 $<15.05$ (W2 S/N $>10$ in COSMOS), which is representative of the shallowest WISE observations, but we will not discuss it in detail. We also include the selection criteria proposed by Assef et al. (2010), which were obtained by simulating WISE photometry using SED models of all objects in SDWFS. For comparison, we also show the numbers for the IRAC-based selection criteria of Lacy et al. (2004, 2007), Stern et al. (2005), and Messias et al. (2012), calculated using the SDWFS photometry. As was done in the previous section, reliability and completeness are measured against the number of objects whose SED fits have $\hat{a}>0.5$.

For the WISE selection methods, regardless of the W2 depth, the most reliable sample is that based on the W1, W2, W3, and W4 selection criteria of Assef et al. (2010), with a 97\% and 98\% reliability for $\mathrm{W} 2<17.11$ and $\mathrm{W} 2<15.73$, respectively. However, because it requires that W4 is detected, it also has the lowest completeness (3\% and $19 \%$ for $\mathrm{W} 2<17.11$ and $\mathrm{W} 2<15.73$, respectively) as measured by the surface density of AGN candidates, with only $44 \mathrm{deg}^{-2}$ candidates with $\mathrm{W} 2<17.11$. Our $R_{90}$ is the second most reliable criterion, with $90 \%$ reliability by design, but it has a much higher completeness, with $53 \%$ for W2 $<15.73$ and $9 \%$ for W2 $<$ 17.11, which translates into AGN candidate surface densities of 117 and $130 \mathrm{deg}^{-2}$, respectively. The four-band criterion of Mateos et al. (2012) also has high reliability, although it is below our $R_{90}$ criterion in both reliability and completeness. The W1, W2, and W3 based selection criteria of both Jarrett et al. (2011) and Mateos et al. (2012) are similar in reliability and completeness, comparable to our $R_{75}$ criterion for $\mathrm{W} 2<15.73$, but somewhat less reliable for $\mathrm{W} 2<17.11$. As discussed by Jarrett et al. (2011), the strength of these criteria are in the deepest WISE fields, where the W1 and W2 depths are below the confusion limit.

With respect to the IRAC-based criteria, the most reliable of those shown are the criteria of Messias et al. (2012), followed by those of Stern et al. (2005). In terms of completeness levels, we note that all criteria shown are similar, except for the highest reliability "KIM" criteria of Messias et al. (2012) based on $K_{s}$, [4.5], [8.0], and MIPS $24 \mu \mathrm{m}$ photometry, which has a lower completeness of $22 \%$ for W2 $<17.11$ and $51 \%$ for $\mathrm{W} 2<15.73$. It is also important to notice that, in principle, completeness and reliability could be improved by further 
Table 3

Surface Density of AGN Candidates at W2 $<15.05$

\begin{tabular}{|c|c|c|c|c|c|}
\hline Selection Criterion & $\begin{array}{c}N \\
\left(\operatorname{deg}^{-2}\right)\end{array}$ & $\begin{array}{c}N(\hat{a}>0.5) \\
\left(\operatorname{deg}^{-2}\right)\end{array}$ & $\begin{array}{c}\text { Reliability } \\
\text { Fraction }\end{array}$ & $\begin{array}{c}\text { Completeness } \\
\text { Fraction }\end{array}$ & $\begin{array}{l}\text { Bands } \\
\text { Used }\end{array}$ \\
\hline \multicolumn{6}{|c|}{ WISE AGN selection } \\
\hline$R_{90}$ & 66 & 59 & 0.90 & 0.77 & $\mathrm{~W} 1, \mathrm{~W} 2$ \\
\hline$R_{75}$ & 87 & 65 & 0.75 & 0.84 & $\mathrm{~W} 1, \mathrm{~W} 2$ \\
\hline$C_{90}$ & 120 & 69 & 0.57 & 0.89 & $\mathrm{~W} 1, \mathrm{~W} 2$ \\
\hline$C_{75}$ & 64 & 59 & 0.93 & 0.77 & $\mathrm{~W} 1, \mathrm{~W} 2$ \\
\hline Paper I & 62 & 58 & 0.94 & 0.75 & $\mathrm{~W} 1, \mathrm{~W} 2$ \\
\hline Jarrett et al. (2011) & 66 & 59 & 0.90 & 0.77 & $\mathrm{~W} 1, \mathrm{~W} 2, \mathrm{~W} 3^{\mathrm{a}}$ \\
\hline Mateos et al. (2012) & 65 & 60 & 0.92 & 0.78 & $\mathrm{~W} 1, \mathrm{~W} 2, \mathrm{~W} 3$ \\
\hline Mateos et al. (2012) & 48 & 39 & 0.82 & 0.51 & $\mathrm{~W} 1, \mathrm{~W} 2, \mathrm{~W} 3, \mathrm{~W} 4$ \\
\hline Assef et al. (2010) & 57 & 55 & 0.96 & 0.71 & $\mathrm{~W} 1, \mathrm{~W} 2$ \\
\hline Assef et al. (2010) & 29 & 29 & 1.00 & 0.37 & $\mathrm{~W} 1, \mathrm{~W} 2, \mathrm{~W} 3, \mathrm{~W} 4$ \\
\hline Wu et al. (2012b) & 99 & 67 & 0.68 & 0.87 & $\mathrm{~W} 1, \mathrm{~W} 2$ \\
\hline \multicolumn{6}{|c|}{ Other infrared AGN selection } \\
\hline Messias et al. (2012) & 67 & 63 & 0.94 & 0.82 & $K_{s},[4.5],[8.0]$ \\
\hline Messias et al. (2012) & 48 & 47 & 0.97 & 0.60 & $K_{s},[4.5],[8.0]$, MIPS $24 \mu \mathrm{m}$ \\
\hline Stern et al. (2005) & 74 & 66 & 0.89 & 0.85 & {$[3.6],[4.5],[5.8],[8.0]$} \\
\hline Lacy et al. (2004) & 123 & 70 & 0.56 & 0.90 & {$[3.6],[4.5],[5.8],[8.0]$} \\
\hline Lacy et al. (2007) & 91 & 68 & 0.75 & 0.89 & {$[3.6],[4.5],[5.8],[8.0]$} \\
\hline
\end{tabular}

Note. ${ }^{a}$ W4 is also used if detected.

adding more information based on other wavelength regimes. Such is the case, for example, with the " $S_{I X}$ " selection scheme of Edelson \& Malkan (2012), which combines WISE, 2MASS, and ROSAT data to identify the brightest AGN in the sky. Including this kind of selection is, however, beyond the scope of this comparison.

\section{PROPERTIES OF WISE AGN CANDIDATES}

In this section, we study the properties of the WISE AGN candidates selected using the criteria developed in the previous section. We first discuss the accuracy to which we can determine photometric redshifts for them. In Section 4.2 we discuss their redshift distribution, and in Section 4.3 we discuss the parameters derived from our SED fitting. In Section 4.4, we present spectroscopic observations of a sample of photometrically selected high-redshift Type 2 AGN candidates.

\subsection{Photometric Redshift Accuracy for WISE AGN Candidates}

Several authors (e.g., Brodwin et al. 2006; Rowan-Robinson et al. 2008; Salvato et al. 2009; Assef et al. 2010) have shown that photometric redshifts of Type 1 AGNs are relatively inaccurate when relying solely on broadband photometry, as is our case. This is mostly due to the lack of strong spectral features that are necessary for anchoring the photometric redshift estimates. Our AGN sample is, however, brighter than those typically studied for photometric redshifts, and has a considerable number of Type 2 AGNs. Photometric redshifts for Type 2 AGNs may be better because the spectral features of the host galaxy are relatively stronger.

We estimate photometric redshifts as discussed in Section 2.3, using, in addition to WISE, all the UV through mid-IR broadband photometry of the field described in Section 2.1. As in Assef et al. (2010), we quantify the photometric redshift accuracy using the statistic

$$
\Delta z=\left[\frac{1}{N} \sum_{i}\left(\frac{z_{\mathrm{phot}}^{i}-z_{\mathrm{spec}}^{i}}{1+z_{\mathrm{spec}}^{i}}\right)^{2}\right]^{1 / 2}
$$

where the index $i$ sums over all objects in a sample and $N$ is their total number. This estimate of the dispersion, however, is typically driven by outliers, so we also estimate $\Delta z_{95}$, the dispersion calculated including only the $95 \%$ of objects with the photometric redshift estimates closest to the spectroscopic estimate.

Panel (a) of Figure 5 shows the spectroscopic and photometric redshifts obtained for the full W2 depth $R_{90}$ AGN candidates, limited to objects with $\hat{a}>0.5$ to be certain we only study the objects of interest. Table 4 shows the dispersion as well as the median offsets for the remaining criteria, again limited to $\hat{a}>0.5$. It also shows the number of AGNs used to compute the statistic and the fraction of objects in every selection criteria that have spectroscopic redshifts. Irrespective of the selection method, the photometric redshifts are fairly inaccurate, with $\Delta z_{95}=0.20-0.23(\Delta z=0.27-0.31)$. This is consistent with the results presented by Assef et al. (2010) for a similar, but fainter, sample of objects. The pile-up of objects at very low $z_{\text {phot }}$ is a degeneracy caused by the galaxy luminosity prior. However, these are only a small part of the sample, and eliminating the prior results in even less accurate estimates for the general population. Panel (b) shows that little is gained in terms of the accuracy when limiting the sample to the brighter W2 S/N $>10$ objects. The same is observed when the sample is further limited by requiring $I<20$, as shown in panel (c).

Considering that photometric redshift estimates for Type 2 AGNs may be more accurate (see above), we further split the bright, final sample (W2 $<15.73$ and $I<20$ and $\hat{a}>0.5$ ) and only investigate objects with considerable obscuration, $E(B-V)>0.5$. We find that $\Delta z_{95}$ drops by $\sim 45 \%$, although $\Delta z$ either decreases only slightly $\left(R_{90}\right.$ and $\left.C_{75}\right)$ or increases 


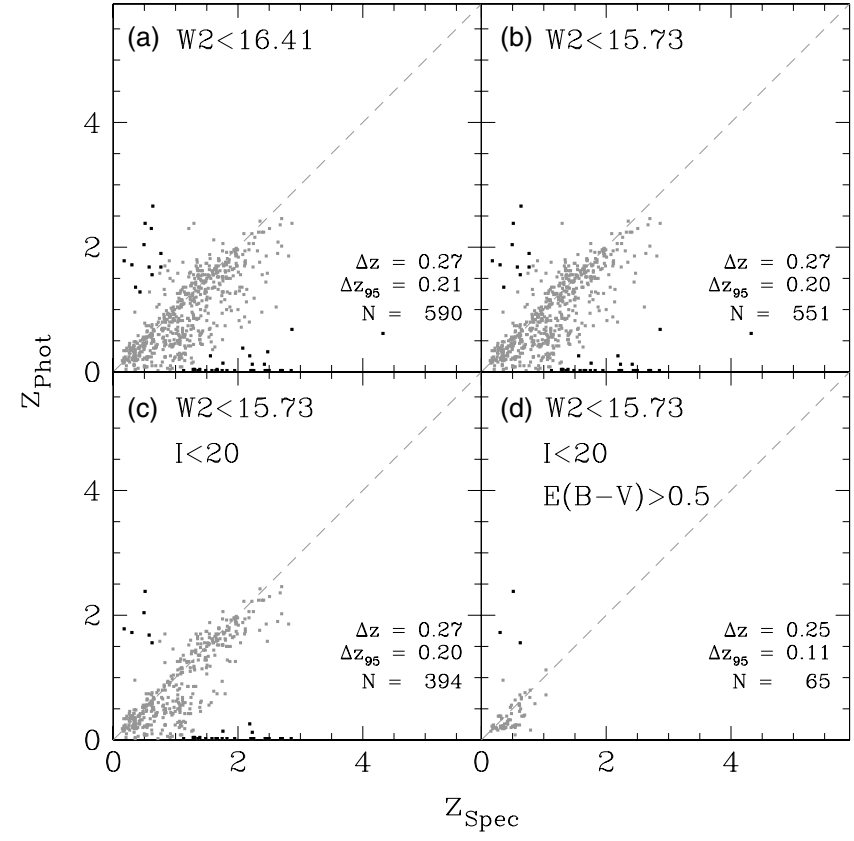

Figure 5. Comparison of photometric and spectroscopic redshifts for the $R_{90}$ sample of AGN candidates for (a) the full W2 depth sample, (b) limited to objects with $\mathrm{W} 2<15.73$, (c) further limited to objects with $I<20$, and (d) even further limited to objects with $E(B-V)>0.5$. Each panel shows the dispersion between the photometric and spectroscopic redshifts for the full sample $(\Delta z)$ and for the $95 \%$ objects with the best estimates to minimize the effect of outliers $\left(\Delta z_{95}\right)$. Black points correspond to objects with $\left|\left(z_{\text {phot }}-z_{\text {spec }}\right) /\left(1+z_{\text {spec }}\right)\right|>0.5$.

$\left(R_{75}\right.$ and $\left.C_{90}\right)$. That these accuracies are still much worse than the $\Delta z_{95} \sim 0.04$ found by Assef et al. (2010) for galaxies of equivalent brightness is most likely due to the sample requirement that $\hat{a}>0.5$, meaning that even though reddened, the accretion disk emission is still likely dominant, or at least significant, in many of the broad bands used.

As mentioned earlier, Assef et al. (2010) showed that although photometric redshifts for AGNs based on broad bands can be inaccurate, the value of $\hat{a}$ obtained from the corresponding SED fit is insensitive to the redshift accuracy, i.e., $\hat{a}$ is relatively independent of photometric redshift. As one of our goals is to study the obscuration in AGNs in a statistically significant manner, we can ask if this holds for the inferred reddening of the AGN component. Hence, we compare the estimates of $E(B-V)$ obtained from the SED fits using the photometric redshift and the spectroscopic redshift estimates. We find that for objects where there is good agreement between $z_{\text {phot }}$ and $z_{\text {spec }}$, the two estimates of $E(B-V)$ are consistent with each other. Unfortunately, however, when the redshift estimates disagree, so do the AGN obscuration estimates, with systematically low $E(B-V)$ values when assuming $z=z_{\text {phot }}$. This will be of particular importance in Section 5.

\subsection{Redshift Distribution of WISE AGN Candidates}

Using the cuts developed in the previous section, we now study the redshift distribution of the different samples of AGN candidates. Although we have a large amount of spectroscopic observations in the Boötes field, we are still missing spectroscopic redshifts for a considerable number of our AGN candidates (see Table 4 for details). For the objects without spectroscopic redshifts, we use the photometric redshift estimates detailed in Section 2.3, although these may not be very accurate (see Section 4.1). We focus on the $R_{90}$ sample, which mitigates this issue as these objects are the ones most likely to have spec-
Table 4

Photometric Redshifts

\begin{tabular}{|c|c|c|c|c|c|c|}
\hline Sample & $\Delta z$ & $\Delta z_{95}$ & $\operatorname{bias} /(1+z)$ & $\operatorname{bias}^{95 \%} /(1+z)$ & $N_{\mathrm{AGN}}$ & $z_{s}$ Fraction \\
\hline \multicolumn{7}{|c|}{ Full W2 depth } \\
\hline$R_{90}$ & 0.27 & 0.20 & 0.15 & 0.14 & 618 & 0.56 \\
\hline$R_{75}$ & 0.29 & 0.21 & 0.14 & 0.13 & 839 & 0.44 \\
\hline$C_{90}$ & 0.31 & 0.23 & 0.13 & 0.12 & 1668 & 0.13 \\
\hline$C_{75}$ & 0.29 & 0.23 & 0.17 & 0.16 & 1360 & 0.10 \\
\hline \multicolumn{7}{|c|}{$\mathrm{W} 2<15.73$} \\
\hline$R_{90}$ & 0.27 & 0.20 & 0.14 & 0.13 & 595 & 0.61 \\
\hline$R_{75}$ & 0.29 & 0.20 & 0.13 & 0.12 & 731 & 0.57 \\
\hline$C_{90}$ & 0.29 & 0.19 & 0.09 & 0.09 & 890 & 0.48 \\
\hline$C_{75}$ & 0.26 & 0.20 & 0.13 & 0.12 & 740 & 0.48 \\
\hline \multicolumn{7}{|c|}{$\mathrm{W} 2<15.73$ and $I<20$} \\
\hline$R_{90}$ & 0.27 & 0.20 & 0.13 & 0.12 & 420 & 0.85 \\
\hline$R_{75}$ & 0.30 & 0.20 & 0.12 & 0.11 & 507 & 0.84 \\
\hline$C_{90}$ & 0.30 & 0.19 & 0.09 & 0.09 & 597 & 0.75 \\
\hline$C_{75}$ & 0.27 & 0.20 & 0.13 & 0.12 & 477 & 0.82 \\
\hline \multicolumn{7}{|c|}{$\mathrm{W} 2<15.73$ and $I<20$ and $E(B-V)>0.5$} \\
\hline$R_{90}$ & 0.25 & 0.11 & 0.10 & 0.11 & 68 & 0.77 \\
\hline$R_{75}$ & 0.42 & 0.12 & 0.09 & 0.10 & 90 & 0.84 \\
\hline$C_{90}$ & 0.42 & 0.11 & 0.04 & 0.05 & 134 & 0.76 \\
\hline$C_{75}$ & 0.24 & 0.12 & 0.11 & 0.12 & 78 & 0.78 \\
\hline
\end{tabular}

Notes. The table shows the measured photometric redshift dispersions $\Delta z$ and $\Delta z_{95}$ (see Section 4.1 for details), as well as the mean bias of each sample, measured as $\left\langle\left|z_{\text {phot }}-z_{\text {spec }}\right|\right\rangle$ for all objects and limited to the $95 \%$ with the best photometric redshift determination. Note that the numbers only reflect the statistics for objects with $\hat{a}>0.5$ to avoid improved accuracies due to contamination by inactive galaxies.

tra from the AGES survey (see Section 2.1 and Kochanek et al. 2012, for details).

Figure 6 shows the redshift distribution of the W2, W1 $\mathrm{S} / \mathrm{N}>3$ depth $R_{90}$ sample. The resulting distribution of AGNs is double peaked, with the main peak at $1 \lesssim z \lesssim 2$ and a smaller peak at $z \sim 0.25$. Almost no objects are at $z \gtrsim 3$. This distribution reflects that WISE has a high sensitivity to obscured AGNs at lower redshifts, where the AGN emission still dominates the observed W1 and W2 fluxes. However, as the redshift increases or the galaxy host contributions become larger, the bias against obscured sources increases. This causes the minimum at $z \sim 0.75$, followed by an increase simply from the increase in comoving volume probed. The W1-W2 color of Type 1 AGN is reddest at $1 \lesssim z \lesssim 2$ (see Figure 1 ) and progressively gets bluer at higher redshift, falling completely out of the selection criteria by $z \sim 3$. A similar behavior is observed for the $R_{75}, C_{75}$, and $C_{90}$ samples, although since contaminants appear preferentially at high redshifts (see Section 3.2), the balance between the peaks for the complete $\hat{a}$ samples is modified. Figure 6 shows that a large number of the $R_{90}$ AGN candidates (56\%) have spectroscopic redshifts. Furthermore, objects lacking spectroscopic redshifts tend to follow a similar photometric redshift distribution, implying that although the uncertainties in the photometric redshifts are very large, they do not seem to systematically bias the distribution. Limiting the samples to only the brighter W2 S/N > 10 objects does not significantly change the shape of the redshift distribution.

\subsection{SED Analysis of WISE AGN Candidates}

A simple way of quantifying the contamination rates in the criteria we have defined is by looking at the best-fit 


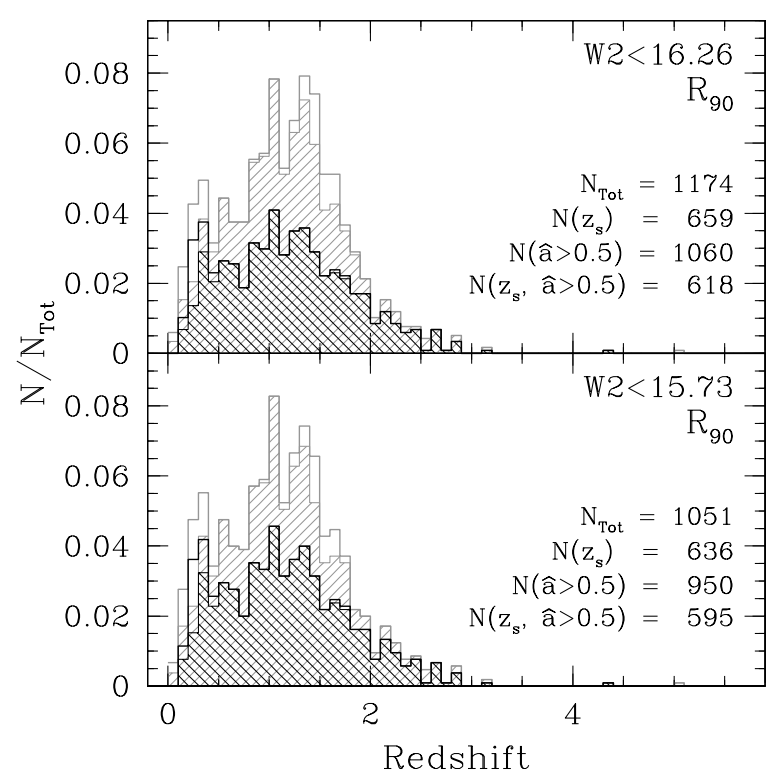

Figure 6. Redshift distribution of the $R_{90}$ sample of AGN candidates for the full W2 depth of the field (top panel) and limited to objects with W2 $<15.73$ (bottom panel). Black histograms show objects with spectroscopic redshifts, while gray histograms add objects with photometric redshift estimates. Shaded histograms only include objects with $\hat{a}>0.5$, while open histograms use objects with all $\hat{a}$ values, including the contaminants.

combination of SED templates to their photometry. The most relevant parameter is $\hat{a}$, defined in Equation (1). As mentioned earlier, this parameter has the useful property of being relatively insensitive to photometric redshift uncertainties (see Assef et al. 2010, for details). Figure 7 shows the distribution of $\hat{a}$ for our full-depth $R_{90}$ and $C_{90} \mathrm{AGN}$ candidate samples. We find that the $R_{90}$ sample is skewed toward objects dominated by their AGN component, with almost no objects being best fit as inactive galaxies. This feature is also observed, although to a somewhat lesser degree, in the $R_{75}$ sample. The $C_{90}$ sample, on the other hand, shows a very considerable peak at $\hat{a}=0$, as expected given its low reliability but high completeness. It also shows, however, a very significant increase in the number of objects with intermediate $\hat{a}$ values. These dominate the distribution for $\hat{a}>0$. Most such objects probably correspond to real AGNs with high host fractions, implying that our reliability optimized criteria are strongly biased against such objects.

It is well known that the luminosity of the spheroidal component of the host galaxy is correlated with the mass of its central $\mathrm{SMBH}$, and that this relation is roughly linear: $L_{\text {host }} \sim M_{\mathrm{BH}}$ (see, e.g., Magorrian et al. 1998; Ferrarese \& Ford 2005; Graham 2007; although also see Graham 2012 for possible deviations). Some authors have postulated that the correlation is also present, and non-evolving, when considering the total host galaxy luminosity instead of just the spheroidal component (Bennert et al. 2010). Regardless, since the Eddington luminosity $L_{\mathrm{Edd}}$ is directly proportional to $M_{\mathrm{BH}}$, the Eddington ratio $\ell_{\mathrm{Edd}}$ can be expressed as

$$
\ell_{\mathrm{Edd}}=\frac{L_{\mathrm{AGN}}}{L_{\mathrm{Edd}}} \sim \frac{L_{\mathrm{AGN}}}{L_{\mathrm{Host}}}=\frac{\hat{a}}{1-\hat{a}} .
$$

Hence, to first order, AGNs whose bolometric output is dominated by the AGN emission (i.e., have high $\hat{a}$ values) also correspond to objects emitting at a high $\ell_{\text {Edd }}$. Similarly, those galaxies for which stellar light represents a higher fraction of their total bolometric output (i.e., low $\hat{a}$ ) are likely radiating

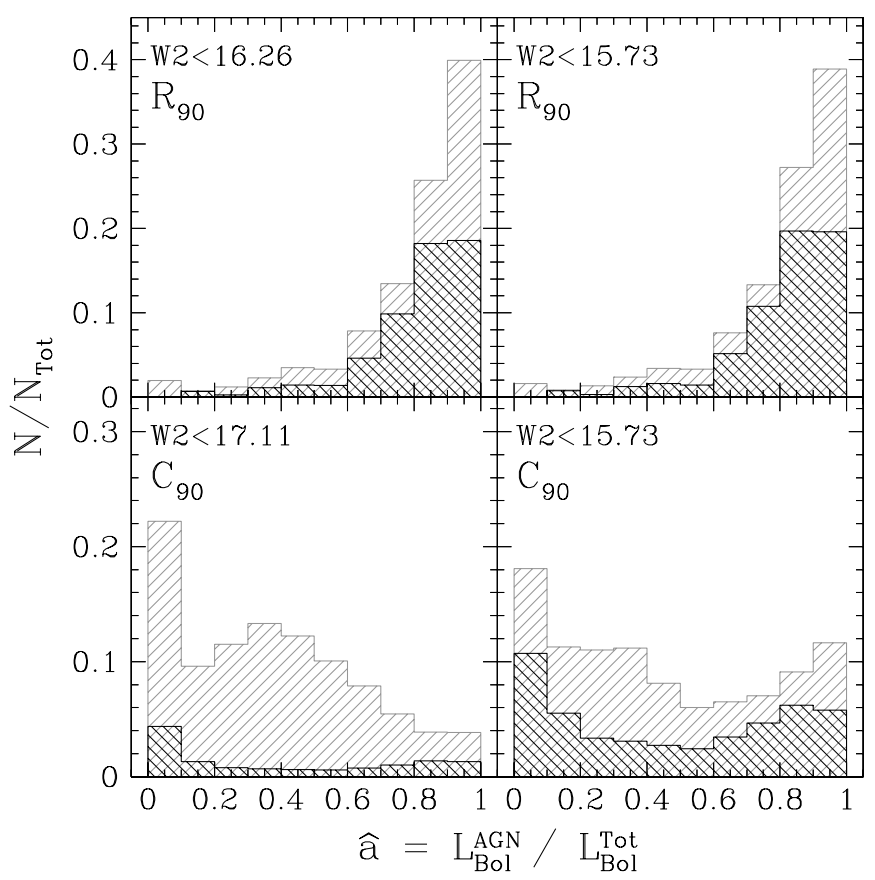

Figure 7. Distribution of $\hat{a}$ values for our $R_{90}$ (top) and $C_{90}$ (bottom) AGN candidate samples. Left panels show the full W2 depth samples, while the right panels are limited to objects with $\mathrm{W} 2<15.73$. Black histograms include only objects with spectroscopic redshifts, while gray histograms also include objects with photometric redshifts.

at lower Eddington ratios. So, in a physical context, we see that our reliability-optimized selection criterion is strongly biased against AGNs radiating at low $\ell_{\text {Edd }}$, but as our selection criterion is shifted to emphasize high completeness, we start recovering them.

Figure 7 also shows the distribution of $\hat{a}$ limited to W2< 15.73. While the $R_{90}$ sample looks nearly the same, the $C_{90}$ sample exhibits a different distribution, with the contamination $(\hat{a}=0$ peak $)$ and the skewness of the distribution shifting to be more similar to the $R_{90}$ sample. Partly, this is because the largest contamination in completeness-optimized samples comes from high-redshift galaxies, which are avoided by the $R_{90}$ criterion. That the peak at high $\hat{a}$ is increased is possibly due to low Eddington ratio AGNs simply being less luminous on average.

The other SED-fit parameter of interest is the amount of obscuration toward the AGN, which is shown in Figure 8 for the $R_{90}$ AGN sample at full and $10 \sigma \mathrm{W} 2$ depths. The most important result to notice is that the WISE AGN selection is sensitive even to objects with high obscuration. In order to interpret the distribution, however, we need to deal with two issues. The first is that because of the algorithm design, the reddening may be slightly underestimated. Second, we need to take into account the selection function of AGES, since the reddening obtained from objects with only photometric redshifts estimates can be inaccurate (Section 4.1). We deal with both issues and present a detailed study of the reddening properties of AGNs in Section 5.

\subsection{Keck Observations}

To highlight the power of WISE in finding highly obscured quasars, we obtained additional spectroscopy of 12 AGN candidates at the Keck Observatory in 2011 April. Since the AGES spectroscopy is limited to $I<22.5$ and is highly complete for $I<21.5$, we emphasized optically fainter candidates which are bright in W2, selected on the basis of an early version of 
Table 5

Summary of Obscured AGN Candidates Observed with Keck/DEIMOS

\begin{tabular}{|c|c|c|c|c|c|c|c|c|c|c|c|}
\hline Name & R.A. & Decl. & $I$ (mag) & W2 (mag) & W1-W2 & $\hat{a}$ & $E(B-V)$ & $z$ & Q & Selection & Notes \\
\hline $\mathrm{W} 1427+3400$ & $14: 27: 54.57$ & $34: 00: 43.31$ & 21.90 & 15.81 & 0.82 & $0.386 \pm 0.091$ & $0.41 \pm 0.11$ & 1.293 & A & $C_{75}$ & [O II $]$ \\
\hline $\mathrm{W} 1427+3403$ & $14: 27: 47.16$ & $34: 03: 41.84$ & 21.24 & 15.76 & 0.50 & $0.209 \pm 0.117$ & $0.33 \pm 0.12$ & 1.137 & A & None & $\mathrm{CaHK}$ \\
\hline W1427+3408 & $14: 27: 17.93$ & $34: 08: 28.60$ & 21.71 & 14.99 & 2.08 & $0.989 \pm 0.009$ & $0.88 \pm 0.04$ & 1.158 & A & $R_{90}$ & {$[\mathrm{O} \mathrm{II}]$} \\
\hline W1428+3359 & $14: 28: 12.31$ & $33: 59: 25.13$ & 23.22 & 15.15 & 1.02 & $0.685 \pm 0.027$ & $0.87 \pm 0.05$ & 1.343 & $\mathrm{~B}$ & $R_{90}$ & [O II $]$ \\
\hline W1429+3529 & $14: 29: 54.83$ & $35: 29: 04.08$ & 21.97 & 15.77 & 0.71 & $0.455 \pm 0.082$ & $0.20 \pm 0.08$ & $(1.3)$ & $\mathrm{F}$ & $C_{90}$ & \\
\hline W1430+3525 & $14: 30: 31.69$ & $35: 25: 17.78$ & 20.64 & 15.07 & 1.54 & $0.944 \pm 0.012$ & $0.45 \pm 0.06$ & 1.106 & A & $R_{90}$ & [O II], CaHK \\
\hline W1430+3530 & 14:30:00.50 & $35: 30: 55.01$ & 19.42 & 15.55 & 1.06 & $0.497 \pm 0.044$ & $0.25 \pm 0.07$ & (1.3) & $\mathrm{F}$ & $R_{75}$ & \\
\hline W1431+3525 & $14: 31: 06.26$ & $35: 25: 46.24$ & 23.63 & 15.60 & 1.88 & $1.000 \pm 0.004$ & $0.78 \pm 0.05$ & (1.3) & $\mathrm{F}$ & $R_{90}$ & {$[\mathrm{O} \mathrm{II}]$} \\
\hline $\mathrm{W} 1431+3528$ & $14: 31: 31.38$ & $35: 28: 38.21$ & 23.62 & 15.47 & 1.77 & $0.990 \pm 0.004$ & $0.89 \pm 0.04$ & 1.343 & A & $R_{90}$ & {$[\mathrm{O} \mathrm{II}]$} \\
\hline W1432+3523 & $14: 32: 23.02$ & $35: 23: 21.41$ & 19.03 & 14.33 & 1.01 & $0.742 \pm 0.006$ & $0.50 \pm 0.03$ & 0.258 & A & $R_{90}$ & $\mathrm{CaHK}, \mathrm{H} \alpha,[\mathrm{N}$ II $]$ \\
\hline W1432+3525 & $14: 32: 37.30$ & $35: 25: 12.56$ & 21.94 & 15.54 & 0.72 & $0.583 \pm 0.036$ & $0.75 \pm 0.05$ & 1.117 & A & $C_{90}$ & Mg II absn, [O II], D4000 \\
\hline W1432+3526 & $14: 32: 22.61$ & $35: 26: 46.88$ & 22.85 & 15.01 & 1.53 & $0.954 \pm 0.006$ & $0.79 \pm 0.05$ & 1.436 & $\mathrm{~B}$ & $R_{90}$ & [O II $]$ \\
\hline
\end{tabular}

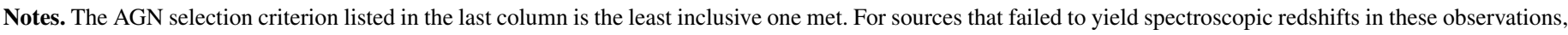
we list the photometric redshift in parentheses. Coordinates are J2000.

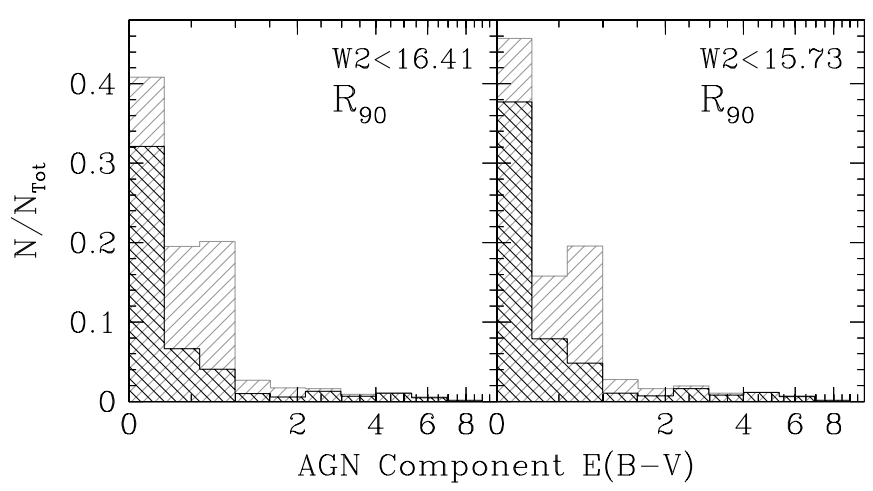

Figure 8. Distribution of best-fit AGN reddening $E(B-V)$ values for our $R_{90}$ AGN candidate samples. The left panel shows the full W2 depth sample, while the right one is limited to objects with $\mathrm{W} 2<15.73$. Black histograms only include objects with spectroscopic redshifts, while gray histograms also include objects with photometric redshifts.

the Paper I criteria. We furthermore required them to not have a measured redshift. Because these observations used a preliminary version of the WISE data, some of the WISE colors and positions changed relative to the more accurate all-sky release. Therefore, the sources we observed have a range of WISE colors and optical magnitudes; based on the WISE all-sky data release, all but one of them are sufficiently red in $\mathrm{W} 1-\mathrm{W} 2$ to be classified as AGN candidates by at least the $C_{90}$ criterion, but not all of them are optically faint $(I>21)$. However, most of the targets do meet the $R_{90}$ selection criterion and prove to be bona fide obscured quasars (see Table 5 for W1-W2 color, W2 magnitude, and AGN classification criteria met by each target).

We observed three Keck slit masks in the Boötes field with the DEep Imaging Multi-Object Spectrograph (DEIMOS; Faber et al. 2003) on UT 2011 April 1-3. We used the $4000 \AA$ orderblocking filter and the $600 \ell \mathrm{mm}^{-1}$ grating (blazed at $7500 \AA$; resolving power $R \equiv \lambda / \Delta \lambda \sim 1600$ for the $1^{\prime \prime} .2$ wide slitlets we employed). We observed a single additional mask using the dualbeam Low Resolution Imaging Spectrometer (LRIS; Oke et al. 1995) on UT 2011 April 28. The LRIS observations employed the $300 \ell \mathrm{mm}^{-1}$ grism on the blue arm of the spectrograph (blazed at $5000 \AA ; R \sim 500$ ), the $400 \ell \mathrm{mm}^{-1}$ grating on the red arm of the spectrograph (blazed at $8500 \AA ; R \sim 700$ ), and $6800 \AA$ dichroic. Data reduction followed standard procedures, and we flux calibrated the data using standard stars from Massey \& Gronwall (1990).
Table 5 summarizes the results for these observations, including measured redshifts, selection criteria, and the best-fit $\hat{a}$ and AGN reddening parameters for the adopted redshift, with errors derived from Monte Carlo re-sampling of the data. Appendix B presents the results for additional Boötes targets observed on these masks. We include the quality ("Q") of each spectroscopic redshift. Quality flag "A" signifies an unambiguous redshift determination, typically relying upon multiple emission or absorption features. Quality flag "B" signifies a less certain redshift determination, such as the robust detection of an isolated emission line, but where the identification of the line is uncertain (e.g., Stern et al. 2000). Quality flag "B" might also be assigned to a source with a robust redshift identification, but where some uncertainty remains as to the astrometric identity of that spectroscopic source. We consider the quality "B" results likely to be correct, but additional spectroscopy would be beneficial. We assign a quality flag " $F$ " to all cases where a spectroscopic redshift could not be reliably determined.

Figure 9 shows the best-fitted SEDs for each of the 8 targets, from the original 12, whose all-sky release WISE $\mathrm{W} 1-\mathrm{W} 2$ colors classify them as AGNs by either the $R_{90}$ or $R_{75}$ criterion. Upon inspecting the optical images, we believe the bright, discrepant $I$-band flux of $\mathrm{W} 1430+3530$ is most likely due to a bright star within $30^{\prime \prime}$ contaminating the photometry. Although most of these objects appear to be real AGNs based on their broadband SEDs, many lack strong, high-ionization lines such as $\mathrm{CIV}, \mathrm{Mg}$ II, and $\mathrm{Ne} \mathrm{V}$, even though lower ionization lines common for star formation are indeed observed (see Table 5). The X-ray community has noticed a related population of X-ray bright, optically normal galaxies (XBONGS; e.g., Civano et al. 2007) where the $X$-ray luminosities require the presence of an actively accreting SMBH while optical spectroscopy reveals an apparently normal, inactive galaxy. Several explanations have been offered to explain such sources, ranging from systematic effects that dilute the AGN signature for the wide slit widths typically used for these distant sources (e.g., Moran et al. 2002), to radiatively inefficient accretion flows (e.g., Trump et al. 2011). Alternatively, at least some of these objects could be better described as AGN-dominated LIRGs or ULIRGs, where the lack of high-ionization emission lines and the red host color may be explained by large-scale obscuration. Some evidence of the $\mathrm{Si}$ $9.7 \mu \mathrm{m}$ absorption feature typical of ULIRGs may be present in a few cases $(\mathrm{W} 1427+3408, \mathrm{~W} 1431+3525$, W1432+3523, and W1432+3526), causing discrepancies between the models and 


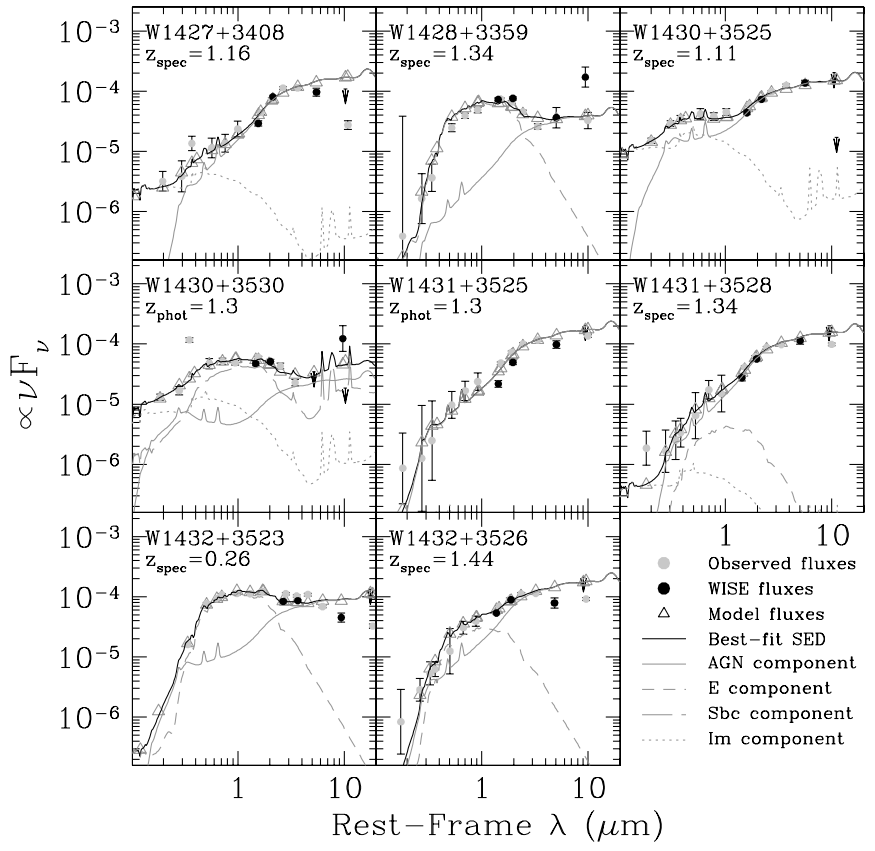

Figure 9. Best-fit SEDs for our sample of highly obscured, high-redshift AGN candidates observed with Keck. Only candidates that met one of the selection criteria beyond the very inclusive $C_{90}$ when using the all-sky data release WISE photometry are shown.

the data, although this feature may also be observed in AGNs under certain conditions (see, e.g., Feltre et al. 2012). Some of the discrepancies observed, however, such as W4 and MIPS $24 \mu \mathrm{m}$ for $\mathrm{W} 1428+3359, \mathrm{~W} 1430+3525$, and $\mathrm{W} 1432+3523$, and W3 for W1432+3526, are possibly simply due to the inherent difficulties of mid-IR observations.

\section{DUST REDDENING IN AGNs}

In this section, we study dust obscuration properties of a set of $362 z<1$ AGNs well detected by WISE with spectroscopic redshifts, $I<20$, and $\hat{a}>0.5$ in the Boötes field. As argued earlier, low-redshift $(z \lesssim 1)$ WISE AGN selection criteria are relatively insensitive to obscuration since they rely on the hot dust emission from the dust torus instead of on the blue colors of the unobscured accretion disk emission, as per optical selection. Hence, we can use WISE to study the properties of dust obscuration in AGNs.

AGN unification models (see, e.g., Antonucci 1993; Urry \& Padovani 1995) propose that Type 1 and Type 2 AGNs are physically equal but are observed at different inclination angles relative to the obscuring material near the AGN. Typically it is assumed that the accretion disk, responsible for the $\lambda \lesssim 1 \mu \mathrm{m}$ continuum emission, extends to radial scales of $\sim 10 \mathrm{AU}$ and is surrounded by highly ionized gas responsible for the broad emission lines. On larger scales $(\sim 1 \mathrm{pc})$ there is dust in the general shape of a "torus" or a flared disk, responsible for the Type 1 /Type 2 dichotomy, that absorbs the optical radiation from the accretion disk and re-emits it in the mid-IR. We refer to this structure as the torus, as is commonly done, although we do not a priori assume a shape for it. The inner edge of the dust torus is determined by where the dust reaches its sublimation temperature due to heating from the accretion disk. Such hot dust produces the emission observed to dominate the mid-IR portion of the AGN SED. Furthermore, narrow emission lines are observed in both Type 1 and Type 2 AGNs and are known not to be polarized (e.g., Antonucci 1993), so the dust structure must be smaller than the narrow-line region $(\sim 1 \mathrm{kpc})$. Given that the Type 1 /Type 2 dichotomy is also manifested in the neutral hydrogen absorption of the X-ray emission, the torus must also be associated with the absorbing gas.

Many properties of the dust torus have been extensively studied. For example, several authors (e.g., Krolik \& Begelman 1988; Nenkova et al. 2002, 2008; Elitzur \& Shlosman 2006; Tristram et al. 2007) have argued that the dust in the torus must be in optically and geometrically thick clumps to reproduce observations, while others (e.g., Dullemond \& van Bemmel 2005; Fritz et al. 2006) argue the dust may be smoothly distributed. A recent study by Feltre et al. (2012), however, suggests that given the same dust composition and the same illuminating source, the difference in the broadband shape of the SEDs from these dust configurations may be too subtle to distinguish between scenarios with current data. In a more global sense, the geometry and evolution of the obscuring structures have also been studied, as, for example, the fraction of obscured objects can have profound implications for explaining the cosmic hard X-ray background (see, e.g., Ueda et al. 2003). Simpson (2005) has shown using the Type 1 and Type 2 AGN optical luminosity function from SDSS that the fraction of Type 2 AGNs increases with decreasing accretion disk luminosity, and a similar behavior has been observed for radio galaxies (Lawrence 1991; Simpson 1998; Grimes et al. 2004) and in the X-rays (e.g., Ueda et al. 2003; Hasinger 2004). Such a behavior can be naturally expected if the scale height $h$ of the obscuring material is independent of (or not linearly related to) the radial size of the structure $\left(R \propto \sqrt{L_{\mathrm{AGN}}}\right)$, such that for brighter AGNs the dust effectively covers a smaller solid angle as viewed from the SMBH. This scenario is usually referred to as the "receding torus model" and was first proposed by Lawrence (1991). In particular, Simpson (2005) has shown that observations appear to be best reproduced if $h \propto L_{\mathrm{AGN}}^{0.23}$.

Combining WISE and all the ancillary observations in the NDWFS Boötes field and performing the SED modeling as detailed in Section 2.3, we can study the average properties of dust obscuration in AGNs by counting the number of objects observed per unit reddening. This approach allows us to quantify the fraction of AGNs that can be classified as Type 1, and also, in principle, to differentiate between different dust geometries and compositions. Note that obscuring dust may also be present in the ISM of the respective host galaxies, and while we expect AGN obscuration to be mainly driven by the dust in the torus and hence delineate the discussion in that direction, we further address galactic-scale obscuration in Section 5.3. Since our sample is, in essence, flux limited, our analysis must properly take into account its selection function. Specifically, it must account for all the biases against highly obscured objects, since higher AGN obscuration can also make the objects appear much fainter depending on the relative contribution and SED shape of the host galaxy. Fortunately, the SED-fitting approach of Assef et al. (2010; see also Section 2.3) is well suited to assess and correct for our survey incompleteness. In the next section, we detail our sample selection function. In Section 5.2, we detail the formalism we use to incorporate the selection function in our measurement of the reddening distribution, while in Section 5.3 we show and discuss the resulting distributions.

\subsection{Sample Selection Function}

To study the reddening distribution, we use a subsample of the larger sample described in Section 2. We require that objects 
have $\mathrm{W} 2<15.73$, a measured spectroscopic redshift such that $E(B-V)$ is accurately estimated (see Section 4.1), and $\hat{a}>0.5$ to minimize possible non-AGN contaminants. Note that we do the initial selection with the SED fits obtained including all the priors described in Section 2.3, which is necessary to ensure all obscured AGNs are real. As discussed there, this can lead to slightly underestimated AGN obscuration. Hence, once the sample is selected, we re-fit the SEDs removing all priors described in Section 5.3 to obtain the final $E(B-V)$ values, although not removing the prior does not qualitatively affect our results. Note, however, that this will cause some incompleteness at the highest obscuration $(E(B-V) \gtrsim 5)$ end of our sample. We visually inspected the SED fit of every source and eliminated 16 galaxies where we believed the AGN classification or the reddening values were spurious due to bad photometry.

We further require that the redshift was determined by AGES, since its well-determined selection function is a crucial component of our analysis. Since many of the objects we consider are extended in the NDWFS imaging and were not necessarily targeted as AGN candidates by the AGES survey, we must also restrict our sample to objects with $I<20$, resulting in a final sample size of 362 AGNs. AGES was designed to ensure subsamples are statistically complete to $I<20$ for galaxies and $I<21.5$ for AGNs (see Kochanek et al. 2012, for details). In order to do this, AGES used a sparse sampling algorithm for galaxies, such that for every defined galaxy subsample, a spectrum was attempted for all objects brighter than a certain magnitude limit and for a percentage (typically 20\%-30\%) of randomly selected fainter galaxies down to a certain magnitude. For example, the main $I$-band selected galaxy sample was observed in full for $I<18.5$ and $20 \%$ of the galaxies were followed in the range $18.5<I<20$. In contrast, there was no sparse sampling for AGN candidates as AGES attempted to get spectra of all of them. Every subsample was assigned a selection code, where $P_{i \text {,sparse }}^{n}$ is the probability that object $i$ of the subsample with selection code $n$ was selected for spectroscopy due to the sparse sampling algorithm.

In addition, the fraction of sources with a successfully measured redshift depends on I-band magnitude. While the survey design minimized the magnitude dependence beyond the sparse sampling, there is still a dependency simply because it is more difficult to obtain redshifts for fainter sources in a fixed integration time. Using the full results of the AGES survey, we estimate for every selection code the fraction of objects for which spectra were attempted and a redshift was measured as a function of $I$-band magnitude, $P_{i, I}^{n}$.

In order to correct for the selection function, we need to estimate for every object the probability that objects with the same optical and IR magnitudes would have been observed, so that we can statistically account for those without spectroscopic observations. Since every object may have been targeted for more than one of the different subsamples, we need to consider the joint probability of all subsamples. Let $P_{i}^{n}=P_{i \text {,sparse }}^{n} \times P_{i, I}^{n}$ and let $N$ be the total number of subsamples object $i$ is part of. We define $C(N, k)$ to be the sum of all possible combinations of $k$ element products of the $P_{i}^{n}$ terms, such that, for example, $C(3,1)=P_{i}^{1}+P_{i}^{2}+P_{i}^{3}, C(3,2)=P_{i}^{1} P_{i}^{2}+P_{i}^{1} P_{i}^{3}+P_{i}^{2} P_{i}^{3}$, and so on. The probability a spectroscopic redshift would have been obtained for objects like object $i$ is then given by

$$
P_{i}=\sum_{k=1}^{N}(-1)^{k+1} C(N, k) .
$$

It can be shown that if any of the terms $P_{i}^{n}=1$, then $P_{i}=1$, as would be expected. For consistency with the original AGES selection, we use the original catalogs of AGES to assess the spectroscopic completeness rather than the catalogs described in Section 2.

\subsection{Method}

In order to incorporate the selection function, we adapt the step-wise maximum-likelihood method (SWML) of Efstathiou et al. (1988). For the remainder of this section, we define $E_{B V} \equiv E(B-V)$. Our goal is to estimate the distribution $\xi\left(E_{B V}\right)=d n / d E_{B V}$, where we remind the reader that $E_{B V}$ corresponds to the reddening only over the AGN component, not over the host galaxy (see Section 2.3 for details). The probability of finding an object with a given reddening $E_{B V}^{i}$ is given by

$$
p_{i} \propto\left(\frac{\xi\left(E_{B V}^{i}\right)}{\int_{0}^{E_{B V, \mathrm{Max}}^{i}} \xi\left(E_{B V}\right) d E_{B V}}\right)^{C_{i}},
$$

where $C_{i}=P_{i}^{-1}$ is calculated using Equation (8) and $E_{B V \text {,Max }}^{i}$ is the maximum reddening object $i$ could have and still be in our sample, which we detail below. We estimate $E_{B V \text {,Max }}$ by varying the reddening of the AGN component of the best-fit combination of SED templates but keeping the amplitude of the components fixed (see Section 2.3 for details).

To apply the SWML method, we discretize the function $\xi\left(E_{B V}\right)$ in bins of $E_{B V}$ rather than assuming a parametric form. We divide $\xi\left(E_{B V}\right)$ into $N_{p}$ bins of value $\xi_{k}$, centered at reddening values $\epsilon_{k}$ with widths $\Delta E_{B V}$. We can then rewrite Equation (9) as

$$
p_{i} \propto\left(\frac{\sum_{k=1}^{N_{p}} W\left(E_{B V}^{i}-\epsilon_{k}\right) \xi_{k}}{\sum_{j=1}^{N_{p}} H\left(\epsilon_{j}-E_{B V, \operatorname{Max}}^{i}\right) \xi_{j} \Delta E_{B V}}\right)^{C_{i}},
$$

where

$$
W(x)= \begin{cases}1 & \text { if }-\Delta E_{B V} / 2 \leqslant x \leqslant \Delta E_{B V} / 2, \\ 0 & \text { otherwise }\end{cases}
$$

and

$$
H(x)= \begin{cases}1 & \text { if } x<-\Delta E_{B V} / 2, \\ \frac{1}{2}-\frac{x}{\Delta E_{B V}} & \text { if }-\Delta E_{B V} / 2 \leqslant x \leqslant \Delta E_{B V} / 2, \\ 0 & \text { if } x>\Delta E_{B V} / 2\end{cases}
$$

The likelihood $\mathcal{L}$ of our sample being drawn from the distribution $\xi\left(E_{B V}\right)$ corresponds to the multiplication of the $p_{i}$ values of all $N_{\mathrm{AGN}}$ objects in our sample. Taking the gradient of $\mathcal{L}$ with respect to $\xi=\left(\xi_{1}, \ldots, \xi_{k}\right)$, we can find that the values that maximize the likelihood are given by

$$
\xi_{k} \Delta E_{B V}=\frac{\sum_{i=1}^{N_{\mathrm{AGN}}} C_{i} W\left(E_{B V}^{i}-\epsilon_{k}\right)}{\sum_{i=1}^{N_{\mathrm{AGN}}} \frac{C_{i} H\left(\epsilon_{k}-E_{B V, \mathrm{Max}}^{i}\right)}{\sum_{j=1}^{N_{p}} H\left(\epsilon_{j}-E_{B V, \mathrm{Max}}^{i}\right) \xi_{j} \Delta E_{B V}}} .
$$

As discussed by Efstathiou et al. (1988), a constraint is needed since the likelihood only depends on the ratios of the $\xi_{k}$ values. We adopt the constraint

$$
g(\xi)=\sum_{k=1}^{N_{p}} \xi_{k}-N_{\mathrm{AGN}},
$$




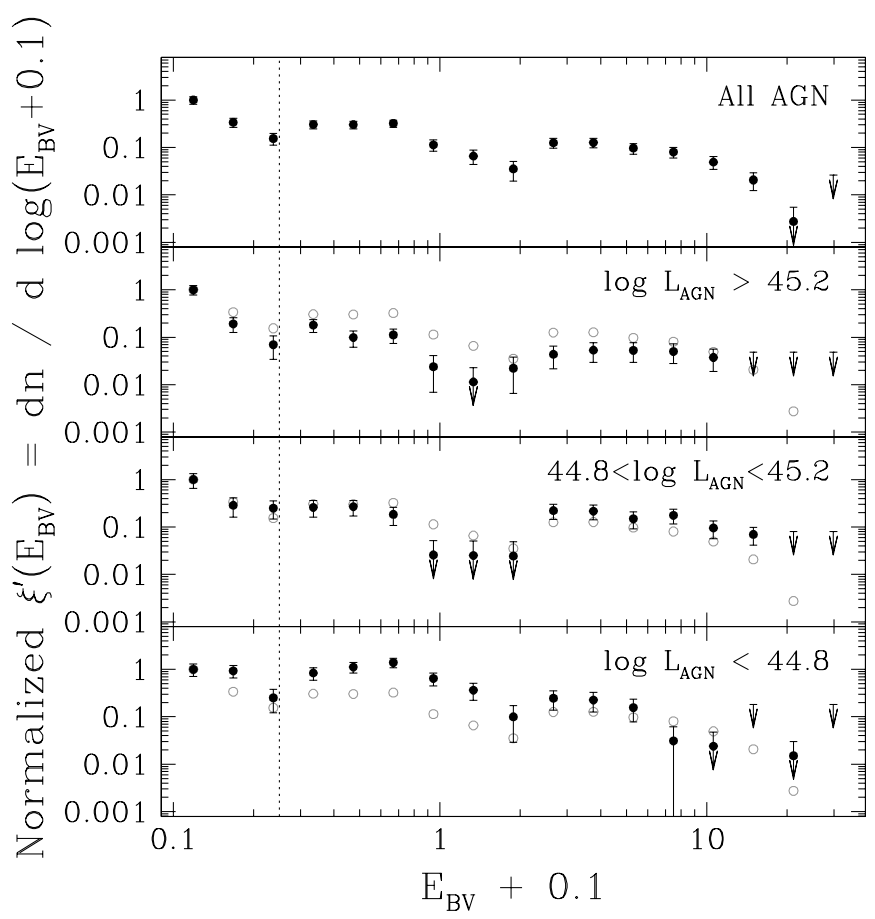

Figure 10. Distribution of reddening values (solid black circles) obtained after using the non-parametric formalism described in Section 5.2 to account for sample incompleteness. For simplicity, we adopt the notation $E_{B V} \equiv E(B-V)$. The top panel shows the distribution obtained when using the complete AGN sample, while the lower three panels show the distributions in three bins of AGN bolometric luminosity ( $\mathrm{erg} \mathrm{s}^{-1}$ ) as defined in Section 2.3. The distribution obtained using all objects is repeated as open gray circles in each of the lower three panels for comparison. All the distributions are normalized to unity in the lowest $E_{B V}$ bin. The vertical dotted line shows our adopted reddening boundary between Type 1 and Type 2 AGNs.

so that the sum of the bins simply equals the number of AGNs, and we maximize $\ln \mathcal{L}^{\prime}=\ln \mathcal{L}+\lambda g(\xi)$, where $\lambda$ is a Lagrange multiplier. Errors in $\xi_{k}$ are estimated using the information matrix as detailed in Efstathiou et al. (1988). In practice, since most of our objects have relatively low reddening values, we prefer to estimate $\xi^{\prime}\left(E_{B V}\right) \equiv d n / d \log \left(E_{B V}+0.1\right)$. Finally, as discussed by Assef et al. (2011), our AGN SED template is as blue as possible, so some of the reddening we find is possibly just due to intrinsic differences in the SEDs of Type 1 AGNs. For example, the mean Type 1 SED template of Richards et al. (2006) is similar to our AGN template with $E(B-V) \approx 0.05$. Since we do not want this to bias the results, we subtract 0.05 from all the $E(B-V)$ values before we construct $\xi$.

\subsection{Results}

Figure 10 shows the distribution of $\xi^{\prime}\left(E_{B V}\right)$ derived using the sample described in Section 5.1. The key thing to note is that the distribution falls with increasing reddening, with a dip at $E(B-V) \sim 2$. A smaller, less significant dip may also be present at $E(B-V) \sim 0.15$. Note that there are no objects observed with a best-fit $E(B-V)>14$. Luminous objects with such high reddening are expected to be rare (see, e.g., Eisenhardt et al. 2012; Wu et al. 2012a; Bridge et al. 2013, for such extreme cases). While the general trend of decreasing numbers with increasing reddening is in all likeliness real, our sample is small enough that the observed dips could in principle be systematic and caused by the non-parametric method we used, as it never imposes the requirement of a smooth distribution. However, when the sample is divided into three luminosity bins with equal numbers of objects, as shown also in Figure 10, the minimum of the dust distribution at $E(B-V) \sim 2$ appears in all of them, further suggesting this feature is real. Assuming that is the case, a few possible explanations are possible.

In the simplest orientation models for AGN unification, most of the obscuration comes from the dust torus. However, if the minimum at intermediate $E(B-V)$ is real, it is unlikely that the dust forms a continuous medium, as it is very hard to have a physically motivated dust distribution that produces such a feature. If the dust is, on the other hand, in geometrically and optically thick clouds, the distribution would simply be the distribution of the obscuration of the clouds convolved with the distribution of inclination angles and covering fractions. However, this is also unlikely to be consistent with a minimum in the distribution at an intermediate obscuration value. Possibly, thick dust clouds are responsible for the $E(B-V) \gtrsim 2$ obscuration, and these are embedded in a diffuse inter-cloud dust medium which is responsible for the lower obscuration part of the $\xi^{\prime}\left(E_{B V}\right)$ distribution.

Alternatively, the two halves of the distribution could be attributed to different sources, with the large obscuration coming from thick dust clouds in a torus-like structure surrounding the AGN, and the lower obscuration coming from diffuse dust in the host galaxy ISM. Naively, one would not expect the distribution of ISM dust obscuration to vary systematically with AGN luminosity. When we divide the sample in three bins of luminosity with equal numbers of objects, as shown in Figure 10, we observe a significantly different shape for the $\xi^{\prime}\left(E_{B V}\right)$ distribution in each bin. We consider this as evidence that the dust obscuration is primarily coming from the vicinity of the AGN and is hence associated with the torus, and we discuss this below in the context of a receding torus. It may be possible that in certain AGN feedback scenarios the column density of the residual dust in the ISM left after the AGN has gone through the blow-out phase (see, e.g., Hopkins et al. 2008) could be related to AGN luminosity during its quasar phase.

Note that since our sample is inherently magnitude limited, we cannot easily disentangle redshift evolution from luminosity evolution. We consider, however, that it is much more likely that the evolution in the dust obscuration is primarily driven by the AGN luminosity since hardly any evolution is observed in the UV through mid-IR SEDs of AGNs with cosmic time (e.g., Richards et al. 2006; Assef et al. 2010). Furthermore, Ueda et al. (2003) have shown that the distribution of neutral gas column densities obscuring the X-ray emission of AGNs is independent of redshift.

We investigate the fraction of Type 1 to Type 2 AGNs by simply adding up the corresponding bins of the $\xi^{\prime}\left(E_{B V}\right)$ distribution. We adopt the standard X-ray boundary of a gas column density of $N_{\mathrm{H}}=10^{22} \mathrm{~cm}^{-2}$ (e.g., Ueda et al. 2003) as the dividing line. Maiolino et al. (2001) have shown that the value of $E(B-V) / N_{\mathrm{H}}$ is significantly below the Galactic value for AGNs and varies significantly among different AGNs. The median value of the Maiolino et al. (2001) sample is $E(B-V) / N_{\mathrm{H}}=1.5 \times 10^{-23} \mathrm{~cm}^{2} \mathrm{mag}$, which puts the Type $1 / 2$ boundary at $E(B-V)=0.15$, or $A_{V}=0.47$ for $R_{V}=3.1$. It also implies that our sample does not contain any Compton-thick AGNs $\left(N_{\mathrm{H}}>10^{24} \mathrm{~cm}^{-2}\right)$, which is a reasonable expectation given the requirement of $\hat{a}>0.5$ and the bias of our method to underestimate this value for the most highly obscured AGNs (see Section 2.3 and Appendix A). From the joint distribution of all AGNs, we find that the fraction of objects that would appear as Type 1 AGNs is $47 \% \pm 8 \%$, consistent with an even split 


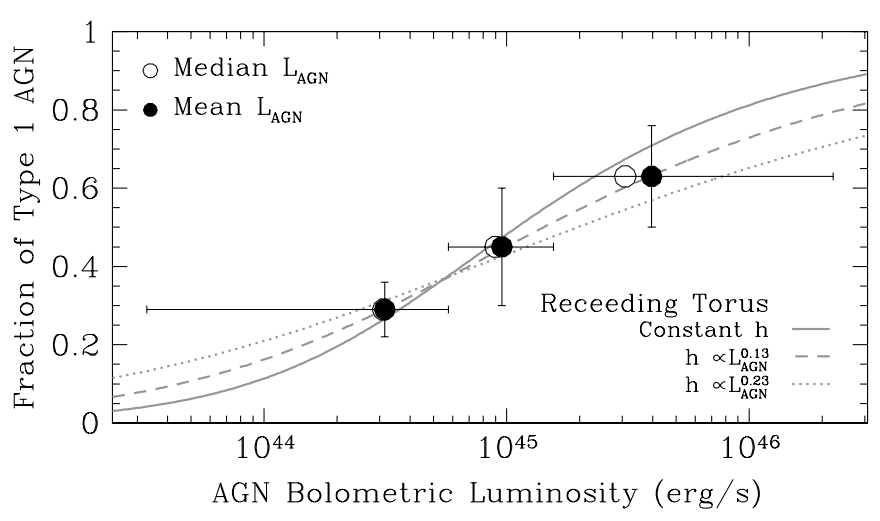

Figure 11. Type $1 \mathrm{AGN}$ fraction as a function of AGN bolometric luminosity, as defined in Section 2.3. The solid points are centered at the mean AGN bolometric luminosity of the bin, while the open ones are centered at the median value. The luminosity error bars show the range of each luminosity bin. The gray lines shows the best-fit receding torus models described in the text.

between the two types. From a purely observational point of view, this is only strictly appropriate for $I<20$ AGNs given our sample selection. However, we note that very little variation in this ratio with $I$-band magnitude is observed in our sample.

Figure 11 shows the fraction of Type 1 AGNs as a function of luminosity when we divide the sample into three luminosity bins with equal numbers of objects per bin. There is a sharp increase in the Type 1 fraction toward higher luminosities. For the lowest luminosity bin, we find that the fraction of objects appearing as Type 1 is $29 \% \pm 7 \%$, increasing to $46 \% \pm 15 \%$ for the intermediate luminosity bin, and to $64 \% \pm 13 \%$ at the highest luminosity. This trend conforms to the idea of a receding torus. Figure 11 compares our observed trends with three different models of a receding torus, taken from Simpson (2005). We model the fraction of Type 1 AGNs by

$$
f_{1}=1-\left[1+3\left(\frac{L_{\mathrm{AGN}}}{L_{\mathrm{AGN}, 0}}\right)^{1-2 \psi}\right]^{-0.5},
$$

which comes from the simple geometry assumed by Simpson (1998) and assuming the scale height $h \propto L_{\mathrm{AGN}}^{\psi}$. At luminosity $L_{\mathrm{AGN}, 0}$, AGNs are evenly split between Types 1 and 2 . We first consider the two cases studied by Simpson (2005), namely, that of a constant $h(\psi=0)$, and his favored scenario of $\psi=0.23$. Note that because Simpson (2005) used [O III] luminosities as proxies for the accretion disk luminosity, we must fit for $L_{\mathrm{AGN}, 0}$, obtaining, respectively, $1.12_{-0.14}^{+0.16} \times 10^{45} \mathrm{erg} \mathrm{s}^{-1}(\psi=0)$ and $1.98_{-0.44}^{+0.57} \times 10^{45} \mathrm{erg} \mathrm{s}^{-1}(\psi=0.23)$. As shown in Figure 11, both of them give a fair representation of the data. If we also fit for the dependence of $h$ on $L_{\mathrm{AGN}}$, we find $\psi=0.13 \pm 0.17$ and $L_{\mathrm{AGN}, 0}=1.42_{-1.26}^{+10.9} \times 10^{45} \mathrm{erg} \mathrm{s}^{-1}$. Unfortunately, our modest sample size does not allow us to more finely sample the Type 1 AGN fraction as a function of AGN luminosity and thereby further constrain such models. We do note, however, that the reddening distributions shown in Figure 10 have significant power to further constrain the dust distribution. This will be further explored in future work.

It is worth noting that Treister et al. (2004) found that a nonevolving Type 1 fraction of $25 \%$ yielded consistency between the soft X-ray and $z$-band flux distributions of AGNs. Given that the AGNs in that study, performed in the GOODS fields, are typically of lower luminosity than the AGN in our sample, this is in general agreement with the $29 \% \pm 7 \%$ we find for our lowest luminosity bin. In contrast, Hopkins et al. (2007) found that an obscured fraction of $0.26\left(L / 10^{46} \mathrm{erg} \mathrm{s}^{-1}\right)^{0.082}$ at rest-frame $4000 \AA$ brings luminosity functions at different wavelengths into good agreement. This value is inconsistent with ours, although a detailed comparison is hard to make as their values are also affected by scatter and luminosity dependence of their assumed bolometric corrections. Further comparison with theoretical expectations to match the hard $\mathrm{X}$-ray background would be useful but is beyond our reach given our insensitivity to Compton-thick AGNs.

\section{CONCLUSIONS}

In an earlier study (Paper I) we used the extensive spectroscopy and photometry of the $2 \mathrm{deg}^{2}$ COSMOS field to study WISE AGN selection. We found that the simple criteria $\mathrm{W} 1-\mathrm{W} 2 \geqslant 0.8$ and $\mathrm{W} 2<15.05$ produce a sample with $95 \%$ reliability and recovered $78 \%$ of the AGNs found with Spitzer IRAC imaging to the same flux depth. Here we have extended this study using the larger $9 \mathrm{deg}^{2}$ NDWFS Boötes field, which has also significantly deeper WISE observations than COSMOS. We show that the reliability of a simple color cut quickly degrades toward fainter fluxes due to the large number of $z \gtrsim 1$ galaxies that contaminate the color selection.

Using the extensive UV through mid-IR broadband photometry available in the NDWFS Boötes field, we have studied W2dependent W1-W2 selection criteria optimized to find AGNs at deeper WISE fluxes than those available in the COSMOS field. We provide different criteria depending on whether the emphasis is on reliability or completeness. We defined a reliabilityoptimized criterion as a W1-W2 color limit that varies as an exponential of $\mathrm{W}^{2}$, where the parameters can be tuned to achieve different reliability levels (Section 3.2). We find that for completeness-optimized selection, no dependence on W2 is needed; a simple W1-W2 color criterion suffices. We find that the criterion of Paper I returns samples with a completeness of approximately $75 \%$.

We have also studied the accuracy of broadband photometric redshifts obtained for the WISE AGN candidates using the Assef et al. (2010) basis of low-resolution SED templates for AGNs and galaxies. We find consistency with the poor accuracy found by previous studies, even though our AGNs are brighter than those typically used in such studies. Furthermore, we find that although the value of the $\hat{a}$ parameter, the luminosity fraction of the AGN with respect to the host plus the AGN, is insensitive to uncertainties in the photometric redshift, the best-fit reddening of the AGN component is strongly affected by those uncertainties. We have studied the distribution of the best-fit $\hat{a}$ parameter of the WISE AGN candidates, showing they are biased toward high values. This means that WISE AGN selection is biased toward objects that are bright with respect to their hosts. Since the luminosity of the host is roughly correlated with the mass of its central SMBH (e.g., Magorrian et al. 1998), this can probably be expressed as a bias toward AGNs radiating at large fractions of their Eddington limits.

Finally, we have studied the distribution of AGNs reddening in the WISE AGN candidates. We have shown that although WISE is more sensitive to unobscured objects, it still finds considerable numbers of highly obscured objects. Extending the sample to include all AGNs found over the field with $\hat{a}>0.5$, spectroscopic redshifts from the AGES survey, and high-S/N WISE W2 fluxes, we have studied the distribution of objects as a function of AGN reddening. We present a formalism based on the SWML method of Efstathiou et al. (1988) designed to 
account for sample incompleteness as a function of obscuration. For a subsample of 362 objects with $I<20$ and W $2<15.73$ for which the selection function is well understood, we find that the reddening distributions depend on AGN bolometric luminosity. The distribution is peaked for unobscured objects and then falls relatively monotonically toward $E(B-V) \sim 2$, raising toward higher values and then dropping again toward $E(B-V) \sim 10$. While it is possible that our small sample size could be driving some of the observed structure, we point out that this shape could be explained by continuous diffuse dust medium in which optically thick dust clouds are embedded. We find that when looking at the complete subsample, $47 \% \pm 8 \%$ of AGNs are Type $1(E(B-V)<0.15$; see Section 5.3). This fraction is a strong function of the AGN bolometric luminosity, consistent with the general scenario of a receding torus. At our lowest luminosity bin, centered at $L_{\mathrm{AGN}}=3 \times 10^{44} \mathrm{erg} \mathrm{s}^{-1}$, we find a Type 1 fraction of $29 \% \pm 7 \%$, which rises to $64 \% \pm 13 \%$ for the highest luminosity bin centered at $L_{\mathrm{AGN}}=4 \times 10^{45} \mathrm{erg} \mathrm{s}^{-1}$. Larger samples, such as that provided by the combination of SDSS and WISE, will provide greater constraints and insight into the dust distribution in AGNs.

We thank M. Dickinson, A.H. Gonzalez, J. Kartaltepe, B. Mobasher, H. Nayyeri, K. Penner, and G. Zeimann for helping us obtain some of the Keck spectroscopic observations used in this work. We thank M. Elitzur for an insightful discussion about dust properties in AGNs. We thank the NDWFS, NEWFIRM, and MAGES survey teams for providing their respective data sets over the Boötes field. We thank the anonymous referee for suggestions that helped improve our work. R.J.A. and C.-W.T. are supported by an appointment to the NASA Postdoctoral Program at the Jet Propulsion Laboratory, administered by Oak Ridge Associated Universities through a contract with NASA. This publication makes use of data products from the Widefield Infrared Survey Explorer, which is a joint project of the University of California, Los Angeles, and the Jet Propulsion Laboratory/California Institute of Technology, funded by the National Aeronautics and Space Administration. Some of the data presented herein were obtained at the W. M. Keck Observatory, which is operated as a scientific partnership among the California Institute of Technology, the University of California, and the National Aeronautics and Space Administration. The Observatory was made possible by the generous financial support of the W. M. Keck Foundation.

\section{APPENDIX A}

\section{ACCURACY OF THE AGN-HOST-GALAXY SED DECOMPOSITION}

A proper characterization of accuracy of the $\hat{a}$ determination in the presence of photometric redshift errors is difficult to quantify beyond the work already presented in Assef et al. (2010), as it depends on several different factors, such as AGN obscuration, "true" redshift of the source, and the intrinsic value of $\hat{a}$. However, we can do a general characterization as follows. We first create a fiducial object with given values of redshift $\left(z_{0}\right)$, AGN fraction $\left(\hat{a}_{0}\right)$, and obscuration $\left(E(B-V)_{0}\right)$, from which we produce a set of photometry in all 18 bands of photometry we use. We assume a W2 magnitude $\mathrm{W} 22_{0}$ and convert into upper bounds all the bands where the fiducial flux is below the corresponding survey limit. Because we want to focus on systematic uncertainties, we assign the synthetic data points uniform error bars, but we do not actually add any random noise.

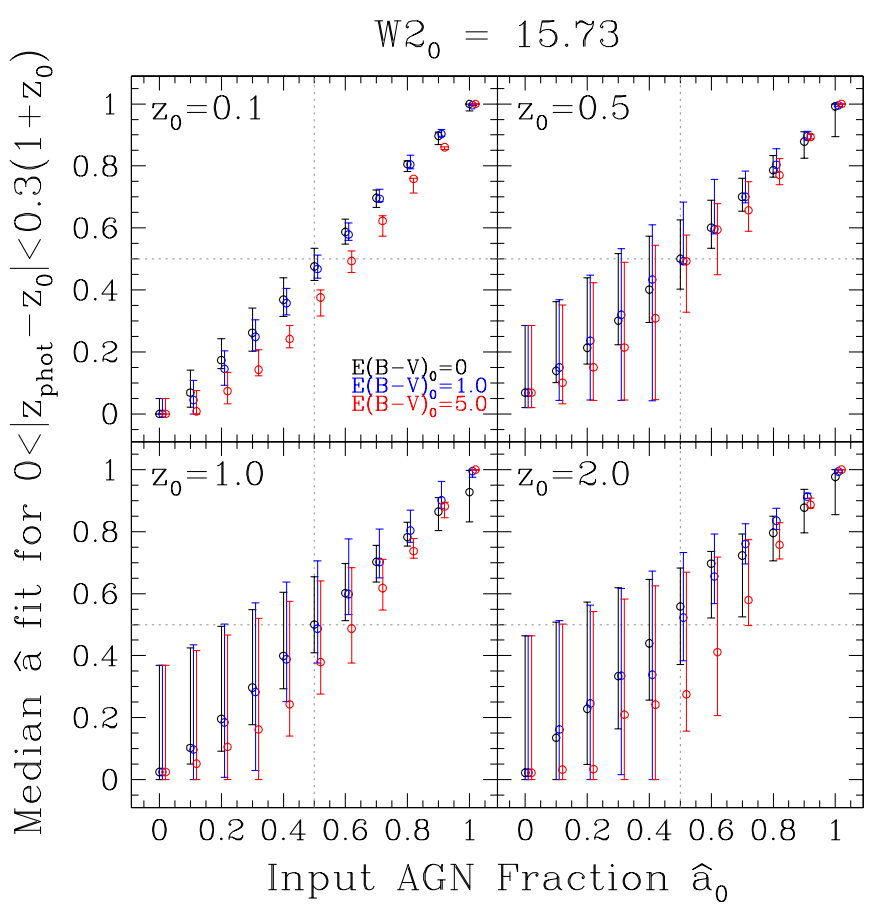

Figure 12. Median $\hat{a}$ values obtained for photometric redshifts within $0.3\left(1+z_{0}\right)$ of the intrinsic redshift $z_{0}$ for a set of simulated galaxies with a given $\hat{a}_{0}$ AGN fraction and $E(B-V)_{0}$ AGN obscuration. We have assigned a W2 $2_{0}$ magnitude of 15.73 for all simulated objects. The error bars show the range encompassing $95.4 \%$ of the trials.

(A color version of this figure is available in the online journal.)

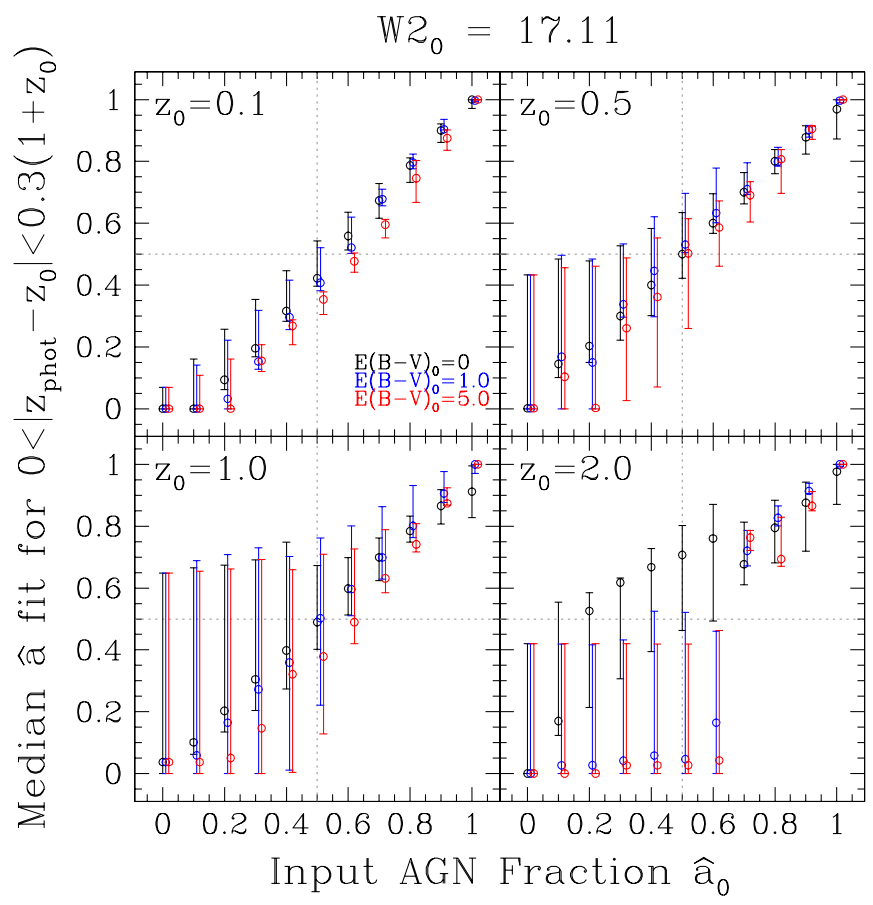

Figure 13. Same as Figure 12, but for simulated objects with an assigned $\mathrm{W} 2{ }_{0}$ magnitude of 17.11 .

(A color version of this figure is available in the online journal.)

We then assume that the photometric redshift estimates have a dispersion of $0.3\left(1+z_{0}\right)$ around $z_{0}$, and proceed to determine $\hat{a}$ in a grid of redshifts covering the whole interval, determining the median and the $95.4 \%$ confidence interval of the obtained values. Finally, we repeat this for different values of $\hat{a}_{0}, E(B-$ $V)_{0}, z_{0}$, and $\mathrm{W} 2_{0}$. The results are presented in Figures 12 and 13. 
Table 6

Additional Results from Keck Observations

\begin{tabular}{lcccccc}
\hline \hline Target Type & R.A. & Decl. & $z$ & Q & Slit Mask(s) & Notes \\
\hline IRAC & $14: 27: 13.39$ & $+34: 09: 04.5$ & 1.343 & A & C[36] & Mg II absorption, [O II] \\
IRAC AGN & $14: 27: 14.32$ & $+34: 09: 01.3$ & 1.692 & B & C[14] & QSO: Mg II (w/ Mg II absorption system at $z=1.342)$ \\
MIPS & $14: 27: 14.63$ & $+34: 08: 46.6$ & 1.343 & B & C[33] & QSO: Mg II \\
MIPS & $14: 27: 14.77$ & $+34: 06: 09.0$ & 1.151 & A & C[28] & Mg II absorption, [O II], D4000 \\
MIPS & $14: 27: 17.40$ & $+34: 07: 26.9$ & 1.082 & A & C[31] & [O II], [Ne III] \\
\hline
\end{tabular}

Notes. Spectroscopic measurements for 129 additional sources. Coordinates shown are in J2000. Q indicates the quality of the redshift (see Section 4.4 for details). Masks A, B, and C were observed with DEIMOS, and the bracketed numbers indicate the DEIMOS slitlet number. Mask F was observed with LRIS.

(This table is available in its entirety in a machine-readable form in the online journal. A portion is shown here for guidance regarding its form and content.)

Note that differences between the two probed photometric depths simply come from the number of bands that have become upper bounds. In general, these figures show that for most parameter combinations, the AGN-host luminosity decomposition is very stable in the presence of these quite large photometric redshift errors. A small bias is observed for the high reddening cases $(E(B-V)=5.0)$ at all $z_{0}$ and $\mathrm{W} 2_{0}$ values, which is simply caused by the weak prior on this quantity discussed above, and completely disappears when we remove it. For $\mathrm{W} 2_{0}=15.73$ (Figure 12), the $\mathrm{S} / \mathrm{N}=10$ limit in Boötes, there is little bias in the median recovered $\hat{a}$ as a function of $\hat{a}_{0}, E(B-V)_{0}$, and $z_{0}$. Error bars become larger only for the most galaxy dominated systems (lowest $\hat{a}_{0}$ ) at $z_{0}=2$, which are exceedingly rare in our sample given our survey depth. For the fainter case of $\mathrm{W} 2_{0}=17.11$ where less bands yield meaningful constraints, the errors are larger, yet the median of the recovered $\hat{a}$ values shows little bias up to $z_{0}=1$. At $z_{0}=2$ significant bias is observed for $\hat{a} \leqslant 0.6$ and $E(B-V)>0$, primarily caused by the lack of constraining information in the UV and optical bands. As mentioned before, however, these systems are extremely rare in our sample and hence will not constitute a significant source of uncertainty in our results.

\section{APPENDIX B}

\section{ADDITIONAL SPECTROSCOPIC REDSHIFTS IN THE NDWFS BOÖTES FIELD}

The four slit masks that we observed were designed to target WISE-selected AGN candidates in the Boötes field, though the low source density of such sources allowed for additional spectroscopic targets. We filled out the masks with (1) IRACselected AGN candidates, using the two-color criteria of Stern et al. (2005) (Column 1 of Table 6, target type = IRAC AGN); (2) $z>1$ galaxy cluster candidates from Eisenhardt et al. (2008) (target type $=$ IRAC cluster); (3) other $4.5 \mu \mathrm{m}$ selected sources from SDWFS, typically selected to have [3.6]-[4.5] $\geqslant$ $-0.1(\mathrm{AB})$, which efficiently selects galaxies at $z>1.2$ (e.g., Galametz et al. 2012) (target type = IRAC); (4) X-ray sources from XBoötes (Murray et al. 2005; Kenter et al. 2005; Brand et al. 2006) (target type $=$ XBoötes); and (5) MIPS $24 \mu \mathrm{m}$ sources in the field (target type $=$ MIPS). Given the interest and use of the Boötes field by a broad community, we include those additional sources here.

Table 6 presents the results for 129 Boötes sources for which we obtained redshifts, including the 11 targeted sources which are also listed in Table 5. The quality flags are defined in Section 4.4. Of particular note is the LRIS mask, which confirms cluster 10.220 from the catalog of Eisenhardt et al. (2008) to be at $z=0.96$.

\section{REFERENCES}

Alonso-Herrero, A., Pérez-González, P. G., Alexander, D. M., et al. 2006, ApJ, 640, 167

Antonucci, R. 1993, ARA\&A, 31, 473

Ashby, M. L. N., Stern, D., Brodwin, M., et al. 2009, ApJ, 701, 428

Assef, R. J., Kochanek, C. S., Ashby, M. L. N., et al. 2011, ApJ, 728, 56

Assef, R. J., Kochanek, C. S., Brodwin, M., et al. 2010, ApJ, 713, 970

Becker, R. H., White, R. L., \& Helfand, D. J. 1995, ApJ, 450, 559

Bennert, V. N., Treu, T., Woo, J.-H., et al. 2010, ApJ, 708, 1507

Brand, K., Brown, M. J. I., Dey, A., et al. 2006, ApJ, 641, 140

Bridge, C. R., Blain, A., Borys, C. J. K., et al. 2013, ApJ, 769, 91

Brodwin, M., Brown, M. J. I., Ashby, M. L. N., et al. 2006, ApJ, 651, 791

Civano, F., Mignoli, M., Comastri, A., et al. 2007, A\&A, 476, 1223

Condon, J. J., Cotton, W. D., Greisen, E. W., et al. 1998, AJ, 115, 1693

Cool, R. J. 2007, ApJS, 169, 21

Croton, D. J., Springel, V., White, S. D. M., et al. 2006, MNRAS, 365, 11 de Vries, W. H., Morganti, R., Röttgering, H. J. A., et al. 2002, AJ, 123, 1784

Donley, J. L., Koekemoer, A. M., Brusa, M., et al. 2012, ApJ, 748, 142

Dullemond, C. P., \& van Bemmel, I. M. 2005, A\&A, 436, 47

Edelson, R., \& Malkan, M. 2012, ApJ, 751, 52

Efstathiou, G., Ellis, R. S., \& Peterson, B. A. 1988, MNRAS, 232, 431

Eisenhardt, P. R., Stern, D., Brodwin, M., et al. 2004, ApJS, 154, 48

Eisenhardt, P. R. M., Brodwin, M., Gonzalez, A. H., et al. 2008, ApJ, 684, 905

Eisenhardt, P. R. M., Wu, J., Tsai, C.-W., et al. 2012, ApJ, 755, 173

Elitzur, M., \& Shlosman, I. 2006, ApJL, 648, L101

Elvis, M., Civano, F., Vignali, C., et al. 2009, ApJS, 184, 158

Elvis, M., Wilkes, B. J., McDowell, J. C., et al. 1994, ApJS, 95, 1

Faber, S. M., Phillips, A. C., Kibrick, R. I., et al. 2003, Proc. SPIE, 4841, 1657

Fabricant, D., Fata, R., Roll, J., et al. 2005, PASP, 117, 1411

Fan, X. 1999, AJ, 117, 2528

Fan, X., Strauss, M. A., Becker, R. H., et al. 2006, AJ, 132, 117

Fazio, G. G., Hora, J. L., Allen, L. E., et al. 2004, ApJS, 154, 10

Feltre, A., Hatziminaoglou, E., Fritz, J., \& Franceschini, A. 2012, MNRAS, 426,120

Ferrarese, L., \& Ford, H. 2005, SSRv, 116, 523

Fritz, J., Franceschini, A., \& Hatziminaoglou, E. 2006, MNRAS, 366, 767

Galametz, A., Stern, D., De Breuck, C., et al. 2012, ApJ, 749, 169

Glikman, E., Bogosavljević, M., Djorgovski, S. G., et al. 2010, ApJ, 710, 1498

Glikman, E., Djorgovski, S. G., Stern, D., et al. 2011, ApJL, 728, L26

Gonzalez, A. H., Brodwin, M., Brown, M. J. I., et al. 2010, in American Astronomical Society Meeting Abstracts, Vol. 216, 415.13

Graham, A. W. 2007, MNRAS, 379, 711

Graham, A. W. 2012, ApJ, 746, 113

Grimes, J. A., Rawlings, S., \& Willott, C. J. 2004, MNRAS, 349, 503

Hasinger, G. 2004, NuPhS, 132, 86

Hopkins, P. F., Hernquist, L., Cox, T. J., \& Kereš, D. 2008, ApJS, 175, 356

Hopkins, P. F., Hernquist, L., Cox, T. J., et al. 2005, ApJ, 630, 705

Hopkins, P. F., Richards, G. T., \& Hernquist, L. 2007, ApJ, 654, 731

Jannuzi, B., Weiner, B., Block, M., et al. 2010, BAAS, 42, 513

Jannuzi, B. T., \& Dey, A. 1999, in ASP Conf. Ser. 193, The Hy-Redshift Universe: Galaxy Formation and Evolution at High Redshift, ed. A. J. Bunker \& W. J. M. van Breugel (San Francisco, CA: ASP), 258

Jarrett, T. H., Cohen, M., Masci, F., et al. 2011, ApJ, 735, 112 
Kenter, A., Murray, S. S., Forman, W. R., et al. 2005, ApJS, 161, 9 Kochanek, C. S., Eisenstein, D. J., Cool, R. J., et al. 2012, ApJS, 200, 8 Krolik, J. H., \& Begelman, M. C. 1988, ApJ, 329, 702

Lacy, M., Petric, A. O., Sajina, A., et al. 2007, AJ, 133, 186

Lacy, M., Storrie-Lombardi, L. J., Sajina, A., et al. 2004, ApJS, 154, 166 Lawrence, A. 1991, MNRAS, 252, 586

Lin, H., Kirshner, R. P., Shectman, S. A., et al. 1996, ApJ, 464, 60 Magorrian, J., Tremaine, S., Richstone, D., et al. 1998, AJ, 115, 2285

Maiolino, R., Marconi, A., Salvati, M., et al. 2001, A\&A, 365, 28

Martin, D. C., Fanson, J., Schiminovich, D., et al. 2005, ApJL, 619, L1

Massey, P., \& Gronwall, C. 1990, ApJ, 358, 344

Mateos, S., Alonso-Herrero, A., Carrera, F. J., et al. 2012, MNRAS, 426, 3271

Messias, H., Afonso, J., Salvato, M., Mobasher, B., \& Hopkins, A. M. 2012, ApJ, 754, 120

Moran, E. C., Filippenko, A. V., \& Chornock, R. 2002, ApJL, 579, L71

Murray, S. S., Kenter, A., Forman, W. R., et al. 2005, ApJS, 161, 1

Nenkova, M., Ivezić, Ž., \& Elitzur, M. 2002, ApJL, 570, L9

Nenkova, M., Sirocky, M. M., Ivezić, Ž., \& Elitzur, M. 2008, ApJ, 685, 147

Oke, J. B., Cohen, J. G., Carr, M., et al. 1995, PASP, 107, 375

Rengelink, R. B., Tang, Y., de Bruyn, A. G., et al. 1997, A\&AS, 124, 259
Richards, G. T., Fan, X., Newberg, H. J., et al. 2002, AJ, 123, 2945

Richards, G. T., Lacy, M., Storrie-Lombardi, L. J., et al. 2006, ApJS, 166, 470 Rowan-Robinson, M., Babbedge, T., Oliver, S., et al. 2008, MNRAS, 386, 697

Salvato, M., Hasinger, G., Ilbert, O., et al. 2009, ApJ, 690, 1250

Shakura, N. I., \& Sunyaev, R. A. 1973, A\&A, 24, 337

Simpson, C. 1998, MNRAS, 297, L39

Simpson, C. 2005, MNRAS, 360, 565

Stengler-Larrea, E. A., Boksenberg, A., Steidel, C. C., et al. 1995, ApJ, 444, 64

Stern, D., Assef, R. J., Benford, D. J., et al. 2012, ApJ, 753, 30

Stern, D., Bunker, A., Spinrad, H., \& Dey, A. 2000, ApJ, 537, 73

Stern, D., Eisenhardt, P., Gorjian, V., et al. 2005, ApJ, 631, 163

Treister, E., Urry, C. M., Chatzichristou, E., et al. 2004, ApJ, 616, 123

Tristram, K. R. W., Meisenheimer, K., Jaffe, W., et al. 2007, A\&A, 474, 837

Trump, J. R., Nagao, T., Ikeda, H., et al. 2011, ApJ, 732, 23

Ueda, Y., Akiyama, M., Ohta, K., \& Miyaji, T. 2003, ApJ, 598, 886

Urry, C. M., \& Padovani, P. 1995, PASP, 107, 803

Wright, E. L., Eisenhardt, P. R. M., Mainzer, A. K., et al. 2010, AJ, 140 , 1868

Wu, J., Tsai, C.-W., Sayers, J., et al. 2012a, ApJ, 756, 96

Wu, X.-B., Hao, G., Jia, Z., Zhang, Y., \& Peng, N. 2012b, AJ, 144, 49 